Michael Hutchings

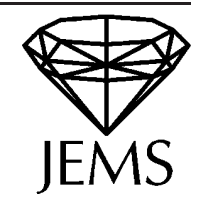

\title{
An index inequality for embedded pseudoholomorphic curves in symplectizations
}

Received November 2, 2000 / final version received December 16, 2001

Published online November 19, 2002 - (C) Springer-Verlag \& EMS 2002

\begin{abstract}
Let $\Sigma$ be a surface with a symplectic form, let $\phi$ be a symplectomorphism of $\Sigma$, and let $Y$ be the mapping torus of $\phi$. We show that the dimensions of moduli spaces of embedded pseudoholomorphic curves in $\mathbb{R} \times Y$, with cylindrical ends asymptotic to periodic orbits of $\phi$ or multiple covers thereof, are bounded from above by an additive relative index. We deduce some compactness results for these moduli spaces.

This paper establishes some of the foundations for a program with Michael Thaddeus, to understand the Seiberg-Witten Floer homology of $Y$ in terms of such pseudoholomorphic curves. Analogues of our results should also hold in three dimensional contact topology.
\end{abstract}

\section{Introduction}

\subsection{Motivation}

Let $\Sigma$ be a compact connected surface, possibly with boundary, with a symplectic form $\omega$. Let $\phi$ be a symplectomorphism of $\Sigma$, and let

$$
Y=\frac{\Sigma \times \mathbb{R}}{(x, t+1) \sim(\phi(x), t)}
$$

be the mapping torus of $\phi$. In this paper we will study the dimensions of moduli spaces of embedded pseudoholomorphic curves in $\mathbb{R} \times Y$, for a suitable almost complex structure. We have two basic motivations for this study.

Our first motivation, in the case $\partial \Sigma=\emptyset$, is to understand the Seiberg-Witten Floer homology of the three-manifold $Y$, an invariant which counts solutions to the Seiberg-Witten equations on the four-manifold $\mathbb{R} \times Y$. Taubes has shown that counting solutions to the Seiberg-Witten equations on a closed symplectic fourmanifold is equivalent to appropriately counting embedded pseudoholomorphic curves in it $[19,20]$. The noncompact 4-manifold $\mathbb{R} \times Y$ has a natural symplectic form, and it is plausible that a version of Taubes's theorem should hold here, relating the Seiberg-Witten Floer homology of $Y$ to a new version of symplectic Floer homology, which we call "periodic Floer homology". This is the homology of a chain complex in which the chains are generated by unions of periodic orbits of

M. Hutchings: 371 Olden Lane, Princeton, NJ 08540, USA e-mail: hutching@ias.edu 
$\phi$ with multiplicity, where hyperbolic orbits cannot have multiplicity greater than one. The differential counts certain pseudoholomorphic curves in $\mathbb{R} \times Y$, which we call "flow lines"; see $\$ 1.2$ for the precise definition. Flow lines need not have genus zero; but they must be embedded, except that there may be repeated "trivial cylinders" which do not intersect any other component. (The precise definition of periodic Floer homology and the conjectured relation with Seiberg-Witten Floer homology are explained in [7]. This relation is also suggested by the approach of Salamon [16] involving symmetric products of $\Sigma$. While Seiberg-Witten Floer homology is defined for closed oriented 3-manifolds, one might also be able to define a version of it for a compact oriented 3-manifold whose boundary is a union of tori, e.g. when $\partial \Sigma \neq \emptyset$, cf. [22].)

Some analytic work is needed to show that periodic Floer homology is well defined. A first step is to compute the dimensions of the moduli spaces of flow lines. This index calculation, which turns out to be rather involved, is the main content of the present paper. In addition to determining the grading of periodic Floer homology, the index theory leads to compactness results for these moduli spaces. The compactness results allow one to define the differential $\delta$ in periodic Floer homology as a certain count of flow lines, and are a step towards proving that $\delta^{2}=0$.

A second motivation for this paper is provided by three-dimensional contact topology. It is interesting to consider embedded pseudoholomorphic curves in the symplectization of a contact 3-manifold, as such curves have found important topological applications in the work of Hofer, Wysocki, and Zehnder. We expect that analogues of our index theorem and compactness will hold in this setting, see $\S 11$. One should further be able to define an analogue of periodic Floer homology on a contact three-manifold. This would be a possibly interesting variant of the symplectic field theory of Eliashberg, Givental, and Hofer [3]. The main difference between periodic Floer homology and symplectic field theory is that the latter theory counts pseudoholomorphic curves that are not necessarily embedded.

\subsection{Flow lines}

We now give the precise definition of the pseudoholomorphic curves that we will study. We begin with their boundary values. A periodic orbit of $\phi$ is a finite set $\gamma$ of points in $\Sigma$ which are cyclically permuted by $\phi$. If $x \in \gamma$ and $p=|\gamma|$ is the period of $\gamma$, the linearized Poincaré return map of $\phi^{p}$ at $x$ is a symplectic linear map $d \phi^{p}: T_{x} \Sigma \rightarrow T_{x} \Sigma$. We say that $\gamma$ is nondegenerate if $1-d \phi^{k p}$ is invertible for all positive integers $k$. Except where stated otherwise, we assume that all periodic orbits of $\phi$ are nondegenerate; this holds for generic $\phi$. We define the Lefschetz sign

$$
(-1)^{\epsilon(\gamma)}=\operatorname{sign} \operatorname{det}\left(1-d \phi^{p}\right) \in\{+1,-1\} .
$$

We say that the orbit $\gamma$ is hyperbolic if $d \phi^{p}$ has real eigenvalues, and elliptic otherwise. The Lefschetz sign is -1 if $\gamma$ is hyperbolic with positive eigenvalues (of $d \phi^{p}$ ); and the Lefschetz sign is +1 if $\gamma$ is elliptic or hyperbolic with negative eigenvalues. 
A periodic orbit $\gamma$ determines an embedded oriented circle in $Y$. More precisely, by equation (1), the mapping torus $Y$ fibers over $S^{1}=\mathbb{R} / \mathbb{Z}$. The flow in the $\mathbb{R}$ direction of $\Sigma \times \mathbb{R}$ induces a vector field on $Y$, which we denote by $\partial_{t}$, and which can also be regarded as a connection on the bundle $Y \rightarrow S^{1}$. Periodic orbits as defined above correspond to embedded closed orbits of the vector field $\partial_{t}$. From now on we will identify periodic orbits with the corresponding circles in $Y$.

Definition 1.1. An orbit set is a finite set of pairs $\alpha=\left\{\left(\alpha_{1}, m_{1}\right), \ldots,\left(\alpha_{k}, m_{k}\right)\right\}$, where $\alpha_{1}, \ldots, \alpha_{k}$ are disjoint periodic orbits, and $m_{1}, \ldots, m_{k}$ are positive integers ( "multiplicities"). The orbit set $\alpha$ is admissible if $m_{i}=1$ whenever $\alpha_{i}$ is hyperbolic.

(Admissible orbit sets generate the chains in periodic Floer homology, and the mod 2 grading is given by the product of the Lefschetz signs; see [7].) If $\alpha_{i}$ has period $p_{i}$, we define the degree of $\alpha$ to be $d=\sum_{i} m_{i} p_{i}$.

To discuss pseudoholomorphic curves, we need to specify an almost complex structure on $\mathbb{R} \times Y$. Let $E$ denote the vertical tangent bundle of $Y \rightarrow S^{1}$. The symplectic form $\omega$ on $\Sigma$ defines a symplectic structure on $E$, and we choose an $\omega$-compatible almost complex structure $J$ on $E$. Compatibility here just means that $J$ respects the orientation, i.e. $\omega(v, J v) \geq 0$. We extend $J$ to an $\mathbb{R}$-invariant almost complex structure on the four-manifold $\mathbb{R} \times Y$, which we also denote by $J$, by specifying that

$$
J\left(\partial_{s}\right)=\partial_{t},
$$

where $s$ denotes the $\mathbb{R}$ coordinate. We call an almost complex structure $J$ on $\mathbb{R} \times Y$ obtained this way admissible. We assume throughout the paper that $J$ is admissible, except in Theorem 1.8(b) and $\$ 9.5$.

We consider pseudoholomorphic curves in $\mathbb{R} \times Y$ of the form $(C, j, u)$, where $C$ is a punctured compact Riemann surface and each component has at least two punctures; $j$ is a complex structure on $C$; and $u: C \rightarrow \mathbb{R} \times Y$ is pseudoholomorphic, $u_{*} \circ j=J \circ u_{*}$. We mod out by reparametrization, i.e. we declare $(C, j, u)$ and $\left(C^{\prime}, j^{\prime}, u^{\prime}\right)$ to be equivalent if there is a diffeomorphism from $C$ to $C^{\prime}$ intertwining $j$ with $j^{\prime}$ and $u$ with $u^{\prime}$. When $u$ is an embedding (or almost an embedding), we identify $(C, j, u)$ with the image of $u$ in $\mathbb{R} \times Y$, which we denote simply by $C$.

If $\gamma$ is a periodic orbit, then $\mathbb{R} \times \gamma$ is a pseudoholomorphic curve, which we call a trivial cylinder. More generally, a pseudoholomorphic curve may have an end smoothly asymptotic as $s \rightarrow+\infty$ to $\mathbb{R} \times \gamma^{k}$, where $s$ denotes the $\mathbb{R}$ coordinate and $\gamma^{k}$ denotes a $k$-fold connected cover of $\gamma$; we call this an outgoing end at $\gamma$ of multiplicity $k$. We call an end asymptotic to $\mathbb{R} \times \gamma^{k}$ as $s \rightarrow-\infty$ an incoming end.

Definition 1.2. A flow line from the orbit set $\left\{\left(\alpha_{i}, m_{i}\right)\right\}$ to the orbit set $\left\{\left(\beta_{j}, n_{j}\right)\right\}$ is a pseudoholomorphic curve $C \subset \mathbb{R} \times Y$ as above, such that:

- $C$ is embedded, except that there may be repeated trivial cylinders, which do not intersect other components of $C$.

- $C$ has outgoing ends at $\alpha_{i}$ with total multiplicity $m_{i}$, incoming ends at $\beta_{j}$ with total multiplicity $n_{j}$, and no other ends.

(The differential in periodic Floer homology is defined by a certain count of flow lines, see [7].) 


\subsection{Some assumptions on $\phi$ and $J$}

In this paper we will usually assume that $\phi$ and $J$ satisfy some additional conditions.

Definition 1.3. Let $\gamma$ be a periodic orbit, of period p, containing $x \in \Sigma$. We say that $(\phi, J)$ is admissible near $\gamma$ if $\gamma$ is nondegenerate and:

(i) There exists a neighborhood $U$ of $x$ in $\Sigma$, and a symplectic identification of $U$ with a subset of $\mathbb{R}^{2}$, sending $x$ to 0 , on which $\phi^{p}$ is linear near 0 .

(ii) The restriction to $E$ of the almost complex structure $J$ on some tubular neighborhood $N$ of $\gamma$ is given by a constant matrix on each fiber of the bundle $N \rightarrow \gamma$ induced by the projection $Y \rightarrow S^{1}$, for a trivialization that is linear with respect to the identication in (i).

Definition 1.4. For a positive integer $d$, we say that $(\phi, J)$ is $d$-admissible if $J$ is admissible and:

(a) $(\phi, J)$ is admissible near all periodic orbits of period $p \leq d$.

(b) Every boundary component of $\Sigma$ has an identification of a neighborhood of it with $(-\epsilon, 0] \times(\mathbb{R} / \mathbb{Z})$ with coordinates $(x, y)$, in which $J\left(\partial_{x}\right)=\partial_{y}$ and $\phi(x, y)=(x, y+\theta)$, where $q \theta \notin \mathbb{Z}$ for all integers $1 \leq q \leq d$.

Condition (a) will simplify the asymptotic analysis of the ends of flow lines, allowing us to focus on the topological calculations. This assumption can probably be removed, but to carry out our proofs without it, one would need to generalize the asymptotic analysis of $[8,1]$. In any case, we can achieve this condition by perturbing $\phi$ and $J$, so this assumption entails no loss of generality for defining periodic Floer homology, see [7].

Condition (b) ensures that a flow line can between orbit sets of degree $d$ can never approach the boundary, by the maximum principle, because on any flow line, $x$ is a harmonic function in a neighborhood of the boundary of $\mathbb{R} \times Y$. Note that if $\theta$ is a rational number with denominator larger than $d$, then there will be degenerate periodic orbits near the boundary of period greater than $d$, but these will not arise in our discussion below.

\subsection{The index inequality}

We will obtain a bound on the dimensions of the moduli spaces of flow lines in terms of a relative index, which we now define.

Let $\alpha=\left\{\left(\alpha_{i}, m_{i}\right)\right\}$ and $\beta=\left\{\left(\beta_{j}, n_{j}\right)\right\}$ be orbit sets. If there are any flow lines from $\alpha$ to $\beta$, then their total homology classes must be equal:

$$
\sum_{i} m_{i}\left[\alpha_{i}\right]=\sum_{j} n_{j}\left[\beta_{j}\right]=h \in H_{1}(Y)
$$

Assuming (2), define $H_{2}(Y ; \alpha, \beta)$ to be the set of relative homology classes of 2-chains $W$ in $Y$ with $\partial W=\sum_{i} m_{i} \alpha_{i}-\sum_{j} n_{j} \beta_{j}$. This is an affine space modelled on $H_{2}(Y)$. If $C$ is a flow line from $\alpha$ to $\beta$, then its projection to $Y$ determines a class $[C] \in H_{2}(Y ; \alpha, \beta)$. 
Definition 1.5. Given $Z \in H_{2}(Y ; \alpha, \beta)$, we define the relative index

$$
\begin{aligned}
I(\alpha, \beta ; Z)= & c_{1}\left(\left.E\right|_{Z}, \tau\right)+Q_{\tau}(Z, Z) \\
& +\sum_{i} \sum_{k=1}^{m_{i}} \mu_{\tau}\left(\alpha_{i}{ }^{k}\right)-\sum_{j} \sum_{k=1}^{n_{j}} \mu_{\tau}\left(\beta_{j}{ }^{k}\right) .
\end{aligned}
$$

Here $\tau$ is a trivialization of $E$ over the $\alpha_{i}$ 's and $\beta_{j}$ 's; $c_{1}$ is the relative first Chern class; $Q_{\tau}$ is a relative intersection pairing; and $\mu_{\tau}$ is the Conley-Zehnder index. These notions are explained in detail in $\$ 2$. The relative index has the following basic properties which are proved in $\S 3$.

\section{Proposition 1.6 (properties of the relative index).}

(a) (Well defined) $I(\alpha, \beta ; Z)$ does not depend on $\tau$.

(b) (Additivity) $I(\alpha, \beta ; Z)+I(\beta, \gamma ; W)=I(\alpha, \gamma ; Z+W)$.

(c) (Parity and Lefschetz signs) If $\alpha$ and $\beta$ are admissible, then

$$
I(\alpha, \beta ; Z) \equiv \sum_{i} \epsilon\left(\alpha_{i}\right)-\sum_{j} \epsilon\left(\beta_{j}\right) \quad \bmod 2 .
$$

(d) (Change of homology class)

$$
I(\alpha, \beta ; Z)-I\left(\alpha, \beta ; Z^{\prime}\right)=\left\langle c(h), Z-Z^{\prime}\right\rangle
$$

where the "index ambiguity class" $c(h)$ is defined by

$$
c(h)=c_{1}(E)+2 \operatorname{PD}(h) \in H^{2}(Y ; \mathbb{Z}) .
$$

Let $C$ be a flow line from $\alpha$ to $\beta$, and let $\mathscr{M}_{C}$ denote the component of the moduli space of flow lines containing $C$. The main result of this paper is the following theorem, which is proved in $\$ 5-\$ 7$.

Theorem 1.7 (index inequality). Let $\alpha$ and $\beta$ be orbit sets of degree $d$. Assume that $(\phi, J)$ is $d$-admissible and $J$ is generic. If $C$ is a flow line from $\alpha$ to $\beta$, then $\mathscr{M}_{C}$ is a manifold and

$$
\operatorname{dim}\left(\mathscr{M}_{C}\right) \leq I(\alpha, \beta ;[C])
$$

Equality holds only if $C$ is admissible in the sense of Definition 4.7.

The index theorem is an inequality, rather than an equality, because the dimension of the moduli space depends on some additional discrete choices. Most importantly, the multiplicities of the outgoing ends at $\alpha_{i}$ determine a partition of the integer $m_{i}$, and the multiplicities of the incoming ends at $\beta_{j}$ determine a partition of $n_{j}$. The dimension depends in part on the genus of $C$, which is determined by a relative adjunction formula; this formula involves the writhes of braids determined by the ends of $C$, which in turn have bounds depending on the above partitions.

For each periodic orbit $\gamma$ and each positive integer $m$, we a priori define two partitions of $m$, the "incoming" and "outgoing" partitions, denoted by $p_{\text {in }}(\gamma, m)$ 
and $p_{\text {out }}(\gamma, m)$, see $\S 4$. If $C$ contains no trivial cylinders, then $C$ is "admissible", i.e. equality can hold in the index theorem, when the partition of $m_{i}$ determined by the outgoing ends at $\alpha_{i}$ agrees with $p_{\text {out }}\left(\alpha_{i}, m_{i}\right)$, and the partition of $n_{j}$ determined by the incoming ends agrees with $p_{\text {in }}\left(\beta_{j}, n_{j}\right)$. When $C$ contains trivial cylinders, the criterion for $C$ to be "admissible" is more complicated, but again is phrased in terms of incoming and outgoing partitions.

\subsection{Compactness}

Theorem 1.7 is strong because the upper bound $I$ on the dimensions of the moduli spaces is additive, by Proposition 1.6(b). This additivity leads to some compactness results which we now state.

If $\alpha$ and $\beta$ are orbit sets and $Z \in H_{2}(Y ; \alpha, \beta)$, let $\mathscr{M}(\alpha, \beta ; Z)$ denote the moduli space of flow lines $C$ from $\alpha$ to $\beta$ with relative homology class $[C]=Z$. Note that $\mathbb{R}$ acts on $\mathscr{M}(\alpha, \beta ; Z)$ by translation in the $\mathbb{R}$ direction of $\mathbb{R} \times Y$. The natural flat connection on $\mathbb{R} \times Y \rightarrow \mathbb{R} \times S^{1}$ allows us to extend the symplectic form $\omega$ on the fibers to a canonical closed 2-form on $\mathbb{R} \times Y$, which we also denote by $\omega$. If $i \in \mathbb{Z}$ and $R \in \mathbb{R}$, define

$$
\mathscr{M}_{i}^{R}(\alpha, \beta)=\bigcup_{\substack{I(\alpha, \beta ; Z)=i \\ \int_{Z} \omega<R}} \mathscr{M}(\alpha, \beta ; Z) / \mathbb{R}
$$

Theorem 1.8 (compactness). Let $\alpha$ and $\beta$ be orbit sets of degree $d$, and assume that $(\phi, J)$ is $d$-admissible and $J$ is generic.

(a) Suppose that $d>\operatorname{genus}(\Sigma)$, or $d=1$, or $\partial \Sigma \neq \emptyset$. Then:

(i) $\mathscr{M}_{1}^{R}(\alpha, \beta)$ is finite for each $R$.

(ii) If $\alpha, \beta$ are admissible, then $\mathscr{M}_{2}^{R}(\alpha, \beta)$ has a compactification $\overline{\mathscr{M}_{2}^{R}}(\alpha, \beta)$ with a natural map

$$
\overline{\mathscr{M}_{2}^{R}}(\alpha, \beta) \backslash \mathscr{M}_{2}^{R}(\alpha, \beta) \longrightarrow \bigcup_{\gamma} \mathscr{M}_{1}^{R}(\alpha, \gamma) \times \mathscr{M}_{1}^{R}(\gamma, \beta)
$$

(b) In general, $J$ can be perturbed to an "almost d-admissible" almost complex structure on $\mathbb{R} \times Y$, see Definition 9.11, such that (i) and (ii) above hold, and the Index theorem 1.7 still holds.

Assertion (i) allows one to define the differential $\delta$ in periodic Floer homology as a certain count of flow lines, and assertion (ii) is a step towards proving that $\delta^{2}=0$, see [7]. (To complete the proof that $\delta^{2}=0$, one needs a gluing theorem to show that for a given $\gamma$, over a pair of flow lines on the right side of (4) with total energy less than $R$, the map (4) is odd-to-one if $\gamma$ is admissible, and even-to-one if $\gamma$ is not admissible, see [7].)

The proof of Theorem 1.8, given in $§ 8-\S 9$, is mostly a standard application of Gromov compactness. The main difficulty is that a priori, sequences of embedded 
pseudoholomorphic curves could converge to non-embedded curves. The hardest part of the proof is to check that sequences of flow lines in the relevant moduli spaces do not converge to multiply covered pseudoholomorphic curves. We will do this by enhancing the index inequality to show that an embedded (or almost embedded) curve underlying such a multiply covered curve would live in a moduli space of negative expected dimension, and hence does not exist for generic $J$. Another problem is that for an admissible almost complex structure, the fibers of the projection $\mathbb{R} \times Y \rightarrow \mathbb{R} \times S^{1}$ are pseudoholomorphic, and these might bubble off as the complex structures on the pseudoholomorphic curves in a sequence degenerate. (A related issue arises in the work of Ionel-Parker [12] on GromovWitten invariants of symplectic sums.) Under the assumption in part (a), we will see that this possibility can be ruled out. If this assumption does not hold, in particular if $g>1$, then the pseudoholomorphic curves corresponding to fibers live in moduli spaces of negative expected dimension. We will then see in the proof of part (b) that an appropriate perturbation of the almost complex structure will make such curves disappear, without interfering with the rest of the proofs of the index theorem and compactness.

\subsection{Other results}

In $\S 10$, as a by-product of the index calculations, we work out a formula for the Euler characteristic of flow lines. This is useful for computing periodic Floer homology in specific examples. In $\S 11$ we make some concluding remarks.

\section{The relative index}

We now give a detailed explanation of the relative index $I$ which appears in the index theorem. We will prove its basic properties (Proposition 1.6) in $\$ 3.3$, after introducing the relative adjunction formulas.

Let $\alpha=\left\{\left(\alpha_{i}, m_{i}\right)\right\}$ and $\beta=\left\{\left(\beta_{j}, n_{j}\right)\right\}$ be orbit sets with the same total homology class (2).

\subsection{Trivializations}

If $\gamma$ is a periodic orbit, let $\mathscr{T}(\gamma)$ denote the set of homotopy classes of symplectic trivializations of $\left.E\right|_{\gamma}$. This is an affine space over $\mathbb{Z}$. We adopt the sign convention that if $\tau_{1}, \tau_{2}:\left.E\right|_{\gamma} \rightarrow S^{1} \times \mathbb{R}^{2}$ are two trivializations, then $\tau_{2}-\tau_{1}$ is the degree of $\tau_{1} \circ \tau_{2}^{-1}: S^{1} \rightarrow \operatorname{Sp}(2, \mathbb{R}) \approx S^{1}$.

We say that a nonvanishing section of $\left.E\right|_{\gamma}$ is $\tau$-trivial if its winding number with respect to $\tau$ is zero.

We define $\mathscr{T}(\alpha, \beta)=\prod_{i} \mathscr{T}\left(\alpha_{i}\right) \times \prod_{j} \mathscr{T}\left(\beta_{j}\right)$. If $\tau \in \mathscr{T}(\alpha, \beta)$, we denote the corresponding elements of $\mathscr{T}\left(\alpha_{i}\right)$ and $\mathscr{T}\left(\beta_{j}\right)$ by $\tau_{i}^{+}$and $\tau_{j}^{-}$. 


\subsection{The relative first Chern class}

If $Z \in H_{2}(Y ; \alpha, \beta)$ and $\tau \in \mathscr{T}(\alpha, \beta)$, we define the relative first Chern class $c_{1}\left(\left.E\right|_{Z}, \tau\right) \in \mathbb{Z}$ as follows: Choose a surface $S$ with boundary and a map $f: S \rightarrow Y$ representing $Z$, choose a $\tau$-trivial (nonvanishing) section $\psi$ of $\left.f^{*} E\right|_{\partial S}$, extend $\psi$ to a generic section of $f^{*} E$ over $S$, and define $c_{1}\left(\left.E\right|_{Z}, \tau\right)$ to be the signed number of zeroes of this extension.

This has the following elementary properties. First, $c_{1}\left(\left.E\right|_{Z}, \tau\right)$ depends only on $Z$ and $\tau$. Second, if we change the homology class $Z$, then

$$
c_{1}\left(\left.E\right|_{Z}, \tau\right)-c_{1}\left(\left.E\right|_{Z^{\prime}}, \tau\right)=\left\langle c_{1}(E), Z-Z^{\prime}\right\rangle
$$

where $c_{1}(E) \in H^{2}(Y ; \mathbb{Z})$ is the ordinary first Chern class. Third, under a change of trivialization, we have

$$
c_{1}\left(\left.E\right|_{Z}, \tau\right)-c_{1}\left(\left.E\right|_{Z}, \tau^{\prime}\right)=\sum_{i} m_{i}\left(\tau_{i}^{\prime+}-\tau_{i}^{+}\right)-\sum_{j} n_{j}\left(\tau_{j}^{\prime-}-\tau_{j}^{-}\right) .
$$

\subsection{The Conley-Zehnder index}

If $\gamma$ is a periodic orbit and $\tau \in \mathscr{T}(\gamma)$, we define the Conley-Zehnder index $\mu_{\tau}(\gamma) \in \mathbb{Z}$ as follows. Recall that there is a natural connection on $Y \rightarrow S^{1}$, whose monodromy is given by $\phi$. The linearized parallel transport induces a connection on $\left.E\right|_{\gamma}$. If we traverse $\gamma$ once, then with respect to the trivialization $\tau$, parallel transport defines a path in $\operatorname{Sp}(2, \mathbb{R})$ from the identity to the linearized return map $d \phi^{p}$, where $p$ is the period of $\gamma$. We define $\mu_{\tau}(\gamma)$ to be the Maslov index of this path of symplectic matrices, see e.g. [17]. Likewise we define $\mu_{\tau}\left(\gamma^{k}\right)$ to be the Maslov index of the path in $\operatorname{Sp}(2, \mathbb{R})$ obtained by traveling $k$ times around $\gamma$. Since we are assuming that all periodic orbits are nondegenerate, these Maslov indices are defined.

The general theory of the Maslov index implies that if we change the trivialization, then

$$
\mu_{\tau}\left(\gamma^{k}\right)-\mu_{\tau^{\prime}}\left(\gamma^{k}\right)=2 k\left(\tau-\tau^{\prime}\right)
$$

Also, the parity of $\mu_{\tau}(\gamma)$ is the opposite of the Lefschetz sign $\epsilon(\gamma)$.

In our two dimensional case, we can explicitly describe the Conley-Zehnder index and its behavior under multiple covers as follows. Fix a nondegenerate periodic orbit $\gamma$ and a trivialization $\tau \in \mathscr{T}(\gamma)$.

\section{Proposition 2.1 (Conley-Zehnder index of multiple covers).}

(a) If $\gamma$ is hyperbolic, then there is an integer $n$ such that

$$
\mu_{\tau}\left(\gamma^{k}\right)=k n
$$

for all $k$. The sign of the eigenvalues $\lambda, \lambda^{-1}$ of the linearized return map $d \phi^{p}$ is $(-1)^{n}$. 
(b) If $\gamma$ is elliptic, then there is an irrational number $\theta$ such that for all $k$,

$$
\mu_{\tau}\left(\gamma^{k}\right)=2\lfloor k \theta\rfloor+1 .
$$

Proof. This can be seen from the explicit picture of $\operatorname{Sp}(2, \mathbb{R})$ given by Segal in [2]. For details see [7].

In case (b), we call $\theta$ the monodromy angle of $\gamma$, and we note that the eigenvalues of the linearized return map are $e^{ \pm 2 \pi i \theta}$. Changing the trivialization $\tau$ will change $\theta$ by an integer. (Perhaps one should refer to $2 \pi \theta$ rather than $\theta$ as the "angle", but this would lead to irritating factors of $2 \pi$ later.)

\subsection{The relative intersection pairing}

Definition 2.2. Let $Z \in H_{2}(Y ; \alpha, \beta)$. A representative of $Z$ is an immersed oriented compact surface $S$ in $[0,1] \times Y$ such that:

- $\partial S$ consists of positively oriented (resp. negatively oriented) covers of $\{1\} \times \alpha_{i}$ (resp. $\left.\{0\} \times \beta_{j}\right)$ whose total multiplicity is $m_{i}\left(\right.$ resp. $\left.n_{j}\right)$.

- $[\pi(S)]=Z$, where $\pi:[0,1] \times Y \rightarrow Y$ denotes the projection.

- $S$ is embedded in $(0,1) \times Y$, and $S$ is transverse to $\{0,1\} \times Y$.

Definition 2.3. If $\tau \in \mathscr{T}(\alpha, \beta)$, we say that $S$ above is a $\tau$-representative of $Z$ if, in addition,

- $\left.\pi\right|_{S}$ is an immersion near $\partial S$.

- $S$ contains $m_{i}$ (resp. $n_{j}$ ) singly covered boundary circles at $\{1\} \times \alpha_{i}$ (resp. $\left.\{0\} \times \beta_{j}\right)$. The $m_{i}$ (resp. $\left.n_{j}\right)$ nonvanishing sections of $E$ over $\alpha_{i}\left(\right.$ resp. $\left.\beta_{j}\right)$, given by projecting the conormal vectors in $S$, are $\tau$-trivial. Moreover, in each fiber of $E$ over $\alpha_{i}$ or $\beta_{j}$, these sections lie in distinct rays (emanating from the origin).

Definition 2.4. If $\tau \in \mathscr{T}(\alpha, \beta)$ and $Z, Z^{\prime} \in H_{2}(Y ; \alpha, \beta)$, we define the relative intersection pairing $Q_{\tau}\left(Z, Z^{\prime}\right) \in \mathbb{Z}$ as follows. Let $S$ and $S^{\prime}$ be $\tau$-representatives of $Z$ and $Z^{\prime}$ such that the projected conormal vectors at the boundary all lie in different rays. Then $Q_{\tau}\left(Z, Z^{\prime}\right)$ is the signed number of intersections of $S$ and $S^{\prime}$ in $(0,1) \times Y$, after perturbing in the interior to make these intersections transverse.

We clearly have the symmetry $Q_{\tau}\left(Z, Z^{\prime}\right)=Q_{\tau}\left(Z^{\prime}, Z\right)$. If we change one of the relative homology classes or the trivialization, then $Q$ behaves as follows.

Lemma 2.5. (a) $Q_{\tau}\left(Z, Z^{\prime}\right)$ is well defined, and

$$
Q_{\tau}\left(Z_{1}, Z^{\prime}\right)-Q_{\tau}\left(Z_{2}, Z^{\prime}\right)=\left(Z_{1}-Z_{2}\right) \cap h .
$$

(b) $Q_{\tau}\left(Z, Z^{\prime}\right)-Q_{\tau^{\prime}}\left(Z, Z^{\prime}\right)=\sum_{i} m_{i}^{2}\left({\tau_{i}^{\prime}}^{+}-\tau_{i}^{+}\right)-\sum_{j} n_{j}^{2}\left(\tau_{j}^{\prime-}-\tau_{j}^{-}\right)$.

Here ' $\cap$ ' indicates homological intersection number in $Y$. 
Proof. (a) Let $S_{1}, S_{2}$, and $S^{\prime}$ be $\tau$-representatives of $Z_{1}, Z_{2}$, and $Z^{\prime}$ respectively. (To show that $Q_{\tau}\left(Z, Z^{\prime}\right)$ is well defined, take $Z_{1}=Z_{2}=Z$.) Then $S_{1} \cup\left(-S_{2}\right)$ is a cycle, which is homologous to

$$
[p t] \otimes\left(Z_{1}-Z_{2}\right) \in H_{2}([0,1] \times Y),
$$

and can be represented by an embedded surface $S^{\prime \prime} \subset\{1 / 2\} \times Y$. If we perturb $S^{\prime}$ to be transverse to $\{1 / 2\} \times Y$ and to $S^{\prime \prime}$, then $S^{\prime} \cap(\{1 / 2\} \times Y)$ is a 1-manifold in the homology class $[p t] \otimes h$. Letting '\#' denote oriented intersection number, we then have

$$
\begin{aligned}
Q_{\tau}\left(Z_{1}, Z^{\prime}\right)-Q_{\tau}\left(Z_{2}, Z^{\prime}\right) & =\#\left(S_{1} \cap S^{\prime}\right)-\#\left(S_{2} \cap S^{\prime}\right) \\
& =\#\left(S^{\prime \prime} \cap S^{\prime}\right) \\
& =\left(Z_{1}-Z_{2}\right) \cap h .
\end{aligned}
$$

In the middle equality above, we have used the fact that the surfaces are $\tau$ representatives, in order to glue $S_{1}$ and $S_{2}$ to a surface in $(0,1) \times Y$ without introducing or cancelling any intersections with $S^{\prime}$ near the boundary.

(b) If $Z=Z^{\prime}$, then this follows from equations (11) and (13), which are proved in $\S 3$. One also needs to use the fact that, as in equation (6),

$$
c_{1}(N, \tau)-c_{1}\left(N, \tau^{\prime}\right)=\sum_{i} m_{i}\left(\tau_{i}^{\prime+}-\tau_{i}^{+}\right)-\sum_{j} n_{j}\left(\tau_{j}^{\prime-}-\tau_{j}^{-}\right) .
$$

To deduce the general case use (a). (One can also prove (b) more directly as in Lemma 8.5.)

\section{Relative adjunction formulas}

We now prove relative adjunction formulas for surfaces with boundary in $[0,1] \times Y$ or with ends in $\mathbb{R} \times Y$. These formulas resemble the adjunction formulas in the closed case, but there is a correction term involving the writhes of braids determined by the ends of the surface. These formulas will play an important role in the subsequent calculations.

\subsection{Braids and writhe}

We begin with some preliminaries on braids. Let $\gamma$ be a closed orbit and $\tau \in \mathscr{T}(\gamma)$. Let $\xi \subset Y$ be a braid in a tubular neighborhood $U$ of $\gamma$. (That is, $\xi$ is a compact embedded 1-manifold, and the projection of $\xi$ to $\gamma$ is a submersion.) Let $A$ denote an annulus, and choose an identification

$$
U \simeq S^{1} \times \mathbb{R}^{2} \simeq A \times(0,1)
$$

whose normal derivative along $\gamma$ agrees with the trivialization $\tau$. We define the writhe $w_{\tau}(\xi) \in \mathbb{Z}$ to be the signed number of crossings in the projection of $\xi$ to $A$. Our sign convention is that counterclockwise twists contribute positively to 
the writhe. (This convention is opposite from much of the knot theory literature, but works well in this paper, especially in $\$ 6$ when we relate writhe to winding numbers.) The writhe depends only on the isotopy class of $\xi$ and the homotopy class of the trivialization $\tau$. If we change the trivialization, then

$$
w_{\tau}(\xi)-w_{\tau^{\prime}}(\xi)=m(m-1)\left(\tau-\tau^{\prime}\right)
$$

where $m$ denotes the number of strands in the braid $\xi$. The reason is that changing the trivialization by one is equivalent to inserting a full counterclockwise twist into the braid, which has writhe $m(m-1)$, as each of the $m$ strands crosses over each of the other $m-1$ strands.

Now let $\alpha$ and $\beta$ be orbit sets and let $S \subset[0,1] \times Y$ be a representative of $Z \in H_{2}(Y ; \alpha, \beta)$, as in Definition 2.2. For $s$ close to 1 , the intersection of $S$ with $\{s\} \times Y$ consists of a braid $\xi_{i}^{+}$with $m_{i}$ strands in a neighborhood of $\alpha_{i}$, for each $i$. Likewise, for $s$ close to 0 we obtain braids $\xi_{j}^{-}$near $\beta_{j}$. To simplify the notation, if $\tau \in \mathscr{T}(\alpha, \beta)$, define the total writhe

$$
w_{\tau}(S)=\sum_{i} w_{\tau_{i}^{+}}\left(\xi_{i}^{+}\right)-\sum_{j} w_{\tau_{j}^{-}}\left(\xi_{j}^{-}\right) .
$$

\subsection{Relative adjunction formulas}

If $C$ is a flow line from $\alpha$ to $\beta$ without trivial cylinders, then under a diffeomorphism of $\mathbb{R}$ with $(0,1)$, we obtain a surface in $(0,1) \times Y$, whose closure in $[0,1] \times Y$ is a representative of $[C]$ as in Definition 2.2.

Proposition 3.1 (relative adjunction formulas). Let $\alpha, \beta$ be orbit sets, let $S$ be a representative of $Z \in H_{2}(Y ; \alpha, \beta)$, and let $\tau \in \mathscr{T}(\alpha, \beta)$. Let $N$ denote the normal bundle to $S$. Then:

(a) If $S$ comes from a flow line without trivial cylinders as above, then

$$
c_{1}\left(\left.E\right|_{Z}, \tau\right)=\chi(S)+c_{1}(N, \tau)
$$

(b) For general representatives $S$, equation (12) holds mod 2, and

$$
c_{1}(N, \tau)=w_{\tau}(S)+Q_{\tau}(Z, Z)
$$

Proof. First note that $c_{1}(N, \tau)$ is well defined, because projection induces a canonical isomorphism $\left.N\right|_{\partial S}=\left.E\right|_{\partial S}$, so $\tau$ induces a trivialization of $\left.N\right|_{\partial S}$.

(a) If $S$ comes from a flow line, then up to a homotopy $(0,1) \approx \mathbb{R}$, we have an isomorphism of complex vector bundles

$$
\left(\left.\underline{\mathbb{C}} \oplus E\right|_{S}\right) \simeq T S \oplus N,
$$

since both are isomorphic to $\left.T(\mathbb{R} \times Y)\right|_{S}$. Let $\psi_{E}$ and $\psi_{N}$ be $\tau$-trivial sections of $\left.E\right|_{\partial S}$ and $\left.N\right|_{\partial S}$, and let $\psi_{S}$ be a nonvanishing section of $\left.T S\right|_{\partial S}$ tangent to $\partial S$. 
Over $\partial S$, under the isomorphism (14), we have a homotopy through nonvanishing sections of the determinant line bundles

$$
1 \wedge \psi_{E} \approx \psi_{S} \wedge \psi_{N}
$$

Now in general, if $L_{i}$ is a complex line bundle on $S$ and $s_{i}$ is a nonvanishing section of $\left.L_{i}\right|_{\partial S}$ up to homotopy for $i=1,2$, then

$$
c_{1}\left(\operatorname{det}\left(L_{1} \oplus L_{2}\right), s_{1} \wedge s_{2}\right)=c_{1}\left(L_{1}, s_{1}\right)+c_{1}\left(L_{2}, s_{2}\right) .
$$

Applying this identity to both sides of (14), with respect to the sections in (15), we obtain (12).

(b) If $S$ does not come from a pseudoholomorphic curve, then the isomorphism (14) still holds at the level of real vector bundles, and still respects the complex structures over $\partial S$ after straightening $S$ to be normal to $\{0,1\} \times Y$. It follows that the relative first Chern classes differ by an even integer, because changing the complex structure on a rank 2 complex vector bundle over a closed surface changes the first Chern class by an even integer. Thus (12) holds mod 2.

To prove (13), let $\epsilon>0$ be small and let $S_{0}=S \cap([\epsilon, 1-\epsilon] \times Y)$. Let $S^{\prime}$ be a surface in which $S \backslash S_{0}$ is replaced by a surface $S_{1}$ consisting of cobordisms with $\tau$-trivial braids, so that $S^{\prime}$ is a $\tau$-representative of $Z$ as in Definition 2.3. Let $\psi$ be a section of the normal bundle $N^{\prime}$ to $S^{\prime}$ that is $\tau$-trivial over $\partial S_{1}$. Let $\psi_{0}, \psi_{1}$ denote the restrictions of $\psi$ to $S_{0}, S_{1}$. We can compute $Q_{\tau}(Z, Z)$ by counting the intersections of $S^{\prime}$ with a pushoff of $S^{\prime}$ via $\psi$, so

$$
\begin{aligned}
Q_{\tau}(Z, Z) & =\# \psi^{-1}(0) \\
& =\# \psi_{0}^{-1}(0)+\# \psi_{1}^{-1}(0) \\
& =c_{1}(N, \tau)+\# \psi_{1}^{-1}(0),
\end{aligned}
$$

where '\#' denotes the number of points with signs, after perturbing to obtain transversality. Now

$$
\# \psi_{1}^{-1}(0)=-w_{\tau}(S) .
$$

The reason is that in our cobordism of braids, we can take $\psi_{1}$ to be the projection of a nonzero vertical tangent vector in $A \times(0,1)$. This section will have zeroes at the branch points of the projection to $([0, \epsilon] \cup[1-\epsilon, 1]) \times Y$, where the writhes of the braids change. After an orientation check, this proves (17). Together with (16), this proves (13).

Remark 3.2 (adjunction with singularities). Suppose $C \rightarrow \mathbb{R} \times Y$ is a pseudoholomorphic curve which is almost a flow line, except that it fails to be embedded at a finite number of singular points. Let $Z=[C]$. Then

$$
c_{1}\left(\left.E\right|_{Z}, \tau\right)=\chi(C)+w_{\tau}(C)+Q_{\tau}(Z, Z)-2 \delta(C) .
$$

Here $\delta(C)$ is a sum of positive integer contributions from each singularity as in [14]. Namely, near each singular point, we can perturb the surface to an immersion which is symplectic with respect to the symplectic form $\omega+d s \wedge d t$ on $\mathbb{R} \times Y$, and which 
has only transverse double point singularities; the local contribution to $\delta$ is then the number of double points. To prove (18), we can carry out the above perturbation near each singularity without affecting any of the terms in (18); then the normal bundle $N \rightarrow C$ is well defined, and a straightforward modification of the proof of Proposition 3.1 shows that (12) still holds, while (13) holds with a correction term of $2 \delta(C)$.

\subsection{Properties of the relative index}

We now prove Proposition 1.6.

Part (a) follows from equations (6) and (7) and Lemma 2.5(b). The key calculation is that $\sum_{k=1}^{m} 2 k=m^{2}+m$.

To prove part (b), we compute $I(\alpha, \beta ; Z)$ and $I(\beta, \gamma ; W)$ using trivializations $\tau_{1} \in \mathscr{T}(\alpha, \beta)$ and $\tau_{2} \in \mathscr{T}(\beta, \gamma)$ which agree over the orbits in $\beta$; and we compute $I(\alpha, \gamma ; Z+W)$ using the corresponding trivialization $\tau_{3} \in \mathscr{T}(\alpha, \gamma)$. Then

$$
\begin{aligned}
c_{1}\left(\left.E\right|_{Z+W}, \tau_{3}\right) & =c_{1}\left(\left.E\right|_{Z}, \tau_{1}\right)+c_{1}\left(\left.E\right|_{W}, \tau_{2}\right), \\
Q_{\tau_{3}}(Z+W, Z+W) & =Q_{\tau_{1}}(Z, Z)+Q_{\tau_{2}}(W, W),
\end{aligned}
$$

and the Conley-Zehnder index terms over $\beta$ cancel.

To prove part (c), we first observe from Proposition 2.1 that if $\alpha$ and $\beta$ are admissible, then

$\sum_{i} \sum_{k=1}^{m_{i}} \mu_{\tau}\left(\alpha_{i}{ }^{k}\right)-\sum_{j} \sum_{k=1}^{n_{j}} \mu_{\tau}\left(\beta_{j}{ }^{k}\right) \equiv \sum_{i}\left(\epsilon\left(\alpha_{i}\right)+m_{i}\right)-\sum_{j}\left(\epsilon\left(\beta_{j}\right)+n_{j}\right) \bmod 2$.

Now let $S$ be a $\tau$-representative of $Z$. Then $w_{\tau}(S)=0$, so by the relative adjunction formulas (12) and (13),

$$
c_{1}\left(\left.E\right|_{Z}, \tau\right)+Q_{\tau}(Z, Z) \equiv \chi(S) \bmod 2 .
$$

By the classification of surfaces, we have

$$
\chi(S) \equiv \sum_{i} m_{i}-\sum_{j} n_{j} \bmod 2 .
$$

Combining the above three equations completes the proof of part (c).

Part (d) follows from equation (5) and Lemma 2.5(a).

\section{Partitions at the ends of flow lines}

As remarked in the introduction, a flow line from $\left\{\left(\alpha_{i}, m_{i}\right)\right\}$ to $\left\{\left(\beta_{j}, n_{j}\right)\right\}$ without trivial cylinders determines partitions of the integers $m_{i}$ and $n_{j}$. In terms of these partitions, we will now define the notion of "admissible flow lines", for which the dimension of the moduli space can be maximized. 


\subsection{Incoming and outgoing partitions}

We begin with the definition of some curious partitions. Let $\theta$ be an irrational number. If $q$ is a positive integer, define

$$
f_{\theta}(q)=q^{-1}\lceil q \theta\rceil .
$$

Let $S_{\theta}$ denote the set of positive integers $q$ such that $f_{\theta}\left(q^{\prime}\right)>f_{\theta}(q)$ for all positive integers $q^{\prime}<q$. Equivalently, $f_{\theta}(q)$ is the smallest rational number larger than $\theta$ with denominator $q$; and $q \in S_{\theta}$ when $\theta$ is approximated from above by a rational number with denominator $q$, more closely than any rational number larger than $\theta$ with denominator less than $q$.

Definition 4.1. If $m$ is a positive integer, the incoming partition $p_{\text {in }}(\theta, m)$ is the partition of $m$ defined inductively as follows: let $r=\max \left(S_{\theta} \cap[1, m]\right)$, and define

$$
p_{\text {in }}(\theta, m)=r \cup p_{\text {in }}(\theta, m-r) .
$$

Note that the use of the symbol ' $U$ ' above is a slight abuse of notation, as a partition may contain the same integer several times.

Remark 4.2. The incoming partition depends only on the class $\theta \bmod 1$. If we vary $\theta$, then $p_{\text {in }}(\theta, m)$ changes only when $\theta$ crosses a rational number with denominator $\leq m$. These facts are clear from the definition.

Definition 4.3. We define the outgoing partition

$$
p_{\text {out }}(\theta, m)=p_{\text {in }}(-\theta, m) \text {. }
$$

Remark 4.4. If $m>1$, then $p_{\text {in }}(\theta, m) \cap p_{\text {out }}(\theta, m)=\emptyset$. (This makes the gluing theory for periodic Floer homology interesting, see [7].)

Proof. In the following, if $\xi$ is a real number, let $\{\xi\} \in[0,1)$ denote its fractional part. Fix $q>1$. If $\{(q-1) \theta\}>\{q \theta\}$, then a calculation shows that $f_{\theta}(q-1) \leq$ $f_{\theta}(q)$, so $q \notin S_{\theta}$. This will happen for $\theta$ or $-\theta$, so

$$
S_{\theta} \cap S_{-\theta}=\{1\} .
$$

One can further show, by induction on the size of the incoming partition, that if $m>1$, then $\{(m-1) \theta\}>\{m \theta\} \Longleftrightarrow 1 \in p_{\text {in }}(\theta, m)$. Applying this observation to $\theta$ and $-\theta$, we obtain

$$
1 \in p_{\text {in }}(\theta, m) \Longleftrightarrow 1 \notin p_{\text {out }}(\theta, m) .
$$

Facts (21) and (22) imply the remark.

The following properties of the incoming partition will be used in $\S 7$ and $\S 9$. We leave the proofs as elementary exercises. 


\begin{tabular}{|c|c|c|c|c|c|c|c|}
\hline & 2 & 3 & 4 & 5 & 6 & 7 & 8 \\
\hline $0,1 / 8$ & \multirow{11}{*}{2} & \multirow{7}{*}{3} & \multirow{5}{*}{4} & \multirow{4}{*}{5} & \multirow{3}{*}{6} & \multirow{2}{*}{7} & 8 \\
\hline $1 / 8,1 / 7$ & & & & & & & 7,1 \\
\hline $1 / 7,1 / 6$ & & & & & & 6,1 & 6,2 \\
\hline $1 / 6,1 / 5$ & & & & & 5,1 & 5,2 & 5,3 \\
\hline $1 / 5,1 / 4$ & & & & 4,1 & 4,2 & 4,3 & 4,4 \\
\hline $1 / 4,2 / 7$ & & & 3.1 & 3.2 & 33 & 7 & 7,1 \\
\hline $2 / 7,1 / 3$ & & & & & & $3,3,1$ & $3,3,2$ \\
\hline $1 / 3,3 / 8$ & & \multirow{4}{*}{2,1} & \multirow{4}{*}{2,2} & \multirow{2}{*}{5} & \multirow{2}{*}{5,1} & \multirow{2}{*}{5,2} & 8 \\
\hline $3 / 8,2 / 5$ & & & & & & & $5,2,1$ \\
\hline $2 / 5,3 / 7$ & & & & \multirow{2}{*}{$2,2,1$} & \multirow{2}{*}{$2,2,2$} & 7 & 7,1 \\
\hline $3 / 7,1 / 2$ & & & & & & $2,2,2,1$ & $2,2,2,2$ \\
\hline $1 / 2,4 / 7$ & \multirow{11}{*}{1,1} & \multirow{4}{*}{3} & \multirow{4}{*}{3,1} & \multirow{2}{*}{5} & \multirow{2}{*}{5,1} & 7 & 7,1 \\
\hline $4 / 7,3 / 5$ & & & & & & $5,1,1$ & 5,3 \\
\hline $3 / 5,5 / 8$ & & & & 3.11 & 33 & 3.3 .1 & 8 \\
\hline $5 / 8,2 / 3$ & & & & $5,1,1$ & 5,3 & $3,3,1$ & $3,3,1,1$ \\
\hline $2 / 3,5 / 7$ & & \multirow{7}{*}{$1,1,1$} & \multirow{2}{*}{4} & \multirow{2}{*}{4,1} & \multirow{2}{*}{$4,1,1$} & 7 & 7,1 \\
\hline $5 / 7,3 / 4$ & & & & & & $4,1,1,1$ & 4,4 \\
\hline $3 / 4,4 / 5$ & & & \multirow{5}{*}{$1,1,1,1$} & 5 & 5,1 & $5,1,1$ & $5,1,1,1$ \\
\hline $4 / 5,5 / 6$ & & & & \multirow{4}{*}{$1, \ldots, 1$} & 6 & 6,1 & $6,1,1$ \\
\hline $5 / 6,6 / 7$ & & & & & \multirow{3}{*}{$1, \ldots, 1$} & 7 & 7,1 \\
\hline $6 / 7,7 / 8$ & & & & & & \multirow{2}{*}{$1, \ldots, 1$} & 8 \\
\hline $7 / 8,1$ & & & & & & & $1, \ldots, 1$ \\
\hline
\end{tabular}

Fig. 1. The incoming partitions for $2 \leq m \leq 8$ and all $\theta$. The left column shows the interval in which $\theta \bmod 1$ lies, and the top row indicates $m$

Lemma 4.5. Let $\theta \in \mathbb{R} \backslash \mathbb{Q}$ and suppose $p_{\text {in }}(\theta, m)=\left(q_{1}, \ldots, q_{k}\right)$. Then:

(a) $\sum_{i=1}^{k}\left\lceil q_{i} \theta\right\rceil=\lceil m \theta\rceil$.

(b) If $i \neq j$, then $\left\lfloor q_{i} \theta\right\rfloor+\left\lfloor q_{j} \theta\right\rfloor<\left\lfloor\left(q_{i}+q_{j}\right) \theta\right\rfloor$.

(c) If $a$ and $b$ are positive integers with $a+b=q_{i}$, then $\lfloor a \theta\rfloor+\lfloor b \theta\rfloor=\left\lfloor q_{i} \theta\right\rfloor$.

Definition 4.6. Let $\gamma$ be a periodic orbit and $m$ a positive integer. We define two partitions of $m$, the incoming partition $p_{\text {in }}(\gamma, m)$ and the outgoing partition $p_{\text {out }}(\gamma, m)$, as follows.

- If $\gamma$ is hyperbolic with positive eigenvalues, then

$$
p_{\text {in }}(\gamma, m)=p_{\text {out }}(\gamma, m)=\{1, \ldots, 1\} .
$$

- If $\gamma$ is hyperbolic with negative eigenvalues, then

$$
p_{\text {in }}(\gamma, m)=p_{\text {out }}(\gamma, m)=\left\{\begin{array}{l}
\{2, \ldots, 2\} \quad \text { if } m \text { is even, } \\
\{2, \ldots, 2,1\} \text { if } m \text { is odd. }
\end{array}\right.
$$

- If $\gamma$ is elliptic with monodromy angle $\theta$ (see $\$ 2.3)$, then $p_{\text {in }}(\gamma, m)=p_{\text {in }}(\theta, m)$ and $p_{\text {out }}(\gamma, m)=p_{\text {out }}(\theta, m)$. 


\subsection{Admissible flow lines}

Let $C$ be a flow line from $\left\{\left(\alpha_{i}, m_{i}\right)\right\}$ to $\left\{\left(\beta_{j}, n_{j}\right)\right\}$. Let $C^{\prime}$ denote the flow line obtained by deleting all trivial cylinders from $C$, and let $m_{i}^{\prime}$ and $n_{j}^{\prime}$ denote the total multiplicities of the ends of $C^{\prime}$ at $\alpha_{i}$ and $\beta_{j}$. The multiplicities of the outgoing ends of $C^{\prime}$ at $\alpha_{i}$ determine a partition of the integer $m_{i}^{\prime}$, which we denote by $p_{i}^{+}$. Likewise the incoming ends of $C^{\prime}$ determine a partition $p_{j}^{-}$of $n_{j}^{\prime}$. The following definition says that $C$ is admissible if these partitions agree with the incoming and outgoing partitions defined in the last section, and if the multiplicities of the trivial cylinders satisfy some additional restrictions.

Definition 4.7. The flow line $C$ is admissible if:

- For each $i$,

$$
p_{i}^{+}=p_{\text {out }}\left(\alpha_{i}, m_{i}^{\prime}\right)
$$

and for each $k$ with $1 \leq k \leq m_{i}-m_{i}^{\prime}$,

$$
p_{\text {out }}\left(\alpha_{i}, m_{i}^{\prime}+k\right)=p_{\text {out }}\left(\alpha_{i}, m_{i}^{\prime}\right) \cup p_{\text {out }}\left(\alpha_{i}, k\right) .
$$

- For each $j$,

$$
p_{j}^{-}=p_{\text {in }}\left(\beta_{j}, n_{j}^{\prime}\right),
$$

and for each $k$ with $1 \leq k \leq n_{j}-n_{j}^{\prime}$,

$$
p_{\text {in }}\left(\beta_{j}, n_{j}^{\prime}+k\right)=p_{\text {in }}\left(\beta_{j}, n_{j}^{\prime}\right) \cup p_{\text {in }}\left(\beta_{j}, k\right) .
$$

Remark 4.8. The trivial cylinder conditions (23) and (24) hold automatically for hyperbolic orbits with positive eigenvalues. If $\alpha_{i}$ is hyperbolic with negative eigenvalues, then (23) asserts that $m_{i}=m_{i}^{\prime}$ when $m_{i}^{\prime}$ is odd.

\section{Index theory}

We now begin to compute the dimension of the moduli space of flow lines and prove the Index theorem 1.7.

\subsection{A general index formula}

We first recall a general index formula for certain $\bar{\partial}$ operators on punctured Riemann surfaces. This formula is proved by Schwarz [18], in different notation.

Let $C$ be a Riemann surface of genus $g$ with $k$ punctures. Let $V \rightarrow C$ be a Hermitian vector bundle of rank $n$ with connection. Let $S$ be a section of $T^{0,1} C \otimes_{\mathbb{C}}$ $\operatorname{End}_{\mathbb{R}}(V)$. Let $p>2$. We are interested in the real linear differential operator

$$
D=\bar{\partial}+S: L_{1}^{p}(V) \rightarrow L^{p}\left(T^{0,1} C \otimes_{\mathbb{C}} V\right)
$$


To make sense of this, we need some assumptions. We choose a holomorphic identification of each of the $k$ ends of $C$ with $\mathbb{R}^{+} \times(\mathbb{R} / \mathbb{Z})$ with coordinates $s, t$, and we choose a trivialization $\tau_{i}$ of $V$ over the $i^{\text {th }}$ end of $C$. In terms of these choices, on the $i^{\text {th }}$ end we can write the operator $D$ as

$$
\partial_{s}+J_{0} \partial_{t}+S_{i}(s, t)
$$

acting on functions $\mathbb{R}^{+} \times S^{1} \rightarrow \mathbb{C}^{n}$. Here $S_{i}(s, t)$ is a real square matrix of rank $2 n$, and $J_{0}$ denotes the standard complex structure on $\mathbb{C}^{n}$. We assume that the limit matrix

$$
S_{i}(t)=\lim _{s \rightarrow \infty} S_{i}(s, t)
$$

exists and is symmetric.

We choose a metric on $C$ which on the ends is asymptotically cylindrical in the above coordinates. This allows us to define the spaces $L_{1}^{p}$ and $L^{p}$ in the definition (25) of $D$ above, and the limiting assumption (27) implies that $D$ sends $L_{1}^{p}$ to $L^{p}$.

Next, define a path of symplectic matrices $\Psi_{i}(t)$ for $t \in[0,1]$ by

$$
\Psi_{i}(0)=1, \quad \frac{d \Psi_{i}(t)}{d t}=J_{0} S_{i}(t) .
$$

We assume that

$$
1 \notin \operatorname{Spec}\left(\Psi_{i}(1)\right)
$$

Then the Maslov index of the path of symplectic matrices $\Psi_{i}$ is defined, and we denote it by $\mu_{i}^{\tau} \in \mathbb{Z}$. Let

$$
c_{1}(V, \tau) \in H^{2}(C, \partial C)=\mathbb{Z}
$$

denote the relative first Chern class of $V$ with respect to the trivializations $\tau_{i}$ at the ends.

Theorem 5.1 (general index formula). [18] Under the assumptions (27) and (28), the operator D is Fredholm, and

$$
\operatorname{ind}(D)=n \chi(C)+2 c_{1}(V, \tau)+\sum_{i=1}^{k} \mu_{i}^{\tau} .
$$

\subsection{Beginning the index computation}

We now use the general index formula to begin the computation of the dimension of the moduli space of flow lines. Let $C$ be a flow line from $\left\{\left(\alpha_{i}, m_{i}\right)\right\}$ to $\left\{\left(\beta_{j}, n_{j}\right)\right\}$, and let $d$ denote the degree of these orbit sets. Assume for now that $C$ contains no trivial cylinders. We write the partitions associated to the ends of $C$ as $p_{i}^{+}=$ $\left(q_{i, 1}, q_{i, 2}, \ldots\right)$ and $p_{j}^{-}=\left(q_{j, 1}^{\prime}, q_{j, 2}^{\prime}, \ldots\right)$. To simplify notation, if $\tau \in \mathscr{T}(\alpha, \beta)$ is 
a trivialization, define

$$
\begin{aligned}
& \mu_{\tau}^{0}(C)=\sum_{i} \sum_{r} \mu_{\tau_{i}^{+}}\left(\alpha_{i}^{q_{i, r}}\right)-\sum_{j} \sum_{r} \mu_{\tau_{j}^{-}}\left(\beta_{j}^{q_{j, r}^{\prime}}\right), \\
& \mu_{\tau}(C)=\sum_{i} \sum_{k=1}^{m_{i}} \mu_{\tau_{i}^{+}}\left(\alpha_{i}^{k}\right)-\sum_{j} \sum_{k=1}^{n_{j}} \mu_{\tau_{j}^{-}}\left(\beta_{j}^{k}\right) .
\end{aligned}
$$

Also let $c_{\tau}(C)=c_{1}\left(\left.E\right|_{[C]}, \tau\right)$ and $Q_{\tau}(C)=Q_{\tau}([C],[C])$.

Lemma 5.2. Assume $(\phi, J)$ is $d$-admissible and $J$ is generic. If $C$ is a flow line without trivial cylinders as above, then $\mathscr{M}_{C}$ is a manifold and

$$
\operatorname{dim}\left(\mathscr{M}_{C}\right) \leq c_{\tau}(C)+Q_{\tau}(C)+w_{\tau}(C)+\mu_{\tau}^{0}(C) .
$$

Proof. A deformation of a flow line might not be embedded, because singularities might appear at the ends. Thus we need to consider "generalized flow lines" (GFL's), see Definition 9.3. Any GFL near $C$ is "quasi-embedded", i.e. embedded except possibly for finitely many singular points, see $\$ 9.3$. Let $\widehat{\mathscr{M}}_{C}$ denote the component of the moduli space of quasi-embedded GFL's containing $C$. A standard transversality calculation, which we defer to Lemma 9.12(b), shows that $\widehat{\mathscr{M}}_{C}$ is a manifold. Then

$$
\operatorname{dim}\left(\widehat{\mathscr{M}}_{C}\right)=\operatorname{ind}\left(D_{C}\right)
$$

where $D_{C}$ denotes the linearized $\bar{\partial}$ operator

$$
D_{C}: L_{1}^{p}(N) \rightarrow L^{p}\left(T^{0,1} C \otimes_{\mathbb{C}} N\right),
$$

see $[15, \S 3]$. Here $p>2$, and $N$ denotes the normal bundle to the embedded curve $C$ in $\mathbb{R} \times Y$. The spaces $L_{1}^{p}$ and $L^{p}$ are defined using an $\mathbb{R}$-invariant metric on $\mathbb{R} \times Y$. Note that as in [20], we use the normal bundle, rather than the restriction of the tangent bundle of $\mathbb{R} \times Y$ to $C$, because we are not fixing the complex structure on the domains of our pseudoholomorphic curves.

We now compute that

$$
\begin{aligned}
\operatorname{ind}\left(D_{C}\right) & =\chi(C)+2 c_{1}(N, \tau)+\mu_{\tau}^{0}(C) \\
& =c_{\tau}(C)+c_{1}(N, \tau)+\mu_{\tau}^{0}(C) \\
& =c_{\tau}(C)+Q_{\tau}(C)+w_{\tau}(C)+\mu_{\tau}^{0}(C) .
\end{aligned}
$$

Here the first equality follows from Theorem 5.1; the operator $D_{C}$ has the local form (26) at the ends of $C$, and the Conley-Zehnder indices come out right, by equations (32) and (34) below. The second and third equalities follow from the relative adjunction formulas (12) and (13).

By equation (18), any flow line $C^{\prime} \in \mathscr{M}_{C}$ satisfies $w_{\tau}\left(C^{\prime}\right)=w_{\tau}(C)$. It follows from this and the discussion in $\S 6$ that $\mathscr{M}_{C}$ is a component of a stratum of $\widehat{\mathscr{M}}_{C}$ in which certain coefficients in asymptotic expansions of the ends vanish. Applying the same transversality calculation as in Lemma 9.12(b) to GFL's satisfying this restriction, we find that $\mathscr{M}_{C}$ is a submanifold of $\widehat{\mathscr{M}}_{C}$ for generic $J$. (But we remark that in the important case when $w_{\tau}(C)$ saturates the upper bound (30) below, equation (18) implies that in fact $\mathscr{M}_{C}=\widehat{\mathscr{M}}_{C}$.) 


\section{Braids at the ends of flow lines}

This section is devoted to proving the following proposition. We use the notation of $\S 5.2$.

Proposition 6.1 (braids). If $C$ is a flow line without trivial cylinders, and if $(\phi, J)$ is admissible near the ends of $C$, then

$$
w_{\tau}(C)+\mu_{\tau}^{0}(C) \leq \mu_{\tau}(C) .
$$

Equality holds only if $C$ is admissible.

The idea of the proof is to show that the braids at the ends of $C$ are iterated nested cablings of torus braids, and to bound their writhe using bounds on winding numbers in terms of the Maslov index from [9]. Proposition 6.1, together with Lemma 5.2, implies Theorem 1.7 for flow lines without trivial cylinders. We will deal with trivial cylinders in $\$ 7$.

\subsection{Asymptotics and winding numbers}

We begin by describing the basic structure of an incoming end of multiplicity $q$ at a periodic orbit $\gamma$. Similar results hold for outgoing ends, but by symmetry we do not need to consider these, see Lemma 6.13.

Assumption 6.2. For the rest of $\S 6$, we assume that $(\phi, J)$ is admissible near $\gamma$.

So we can choose a neighborhood $N$ of $\gamma$ and an identification

$$
N \simeq S^{1} \times \mathbb{R}^{2}
$$

identifying $\gamma \simeq S^{1} \times\{0\}$, compatible with the projection $N \rightarrow \gamma$ induced by the projection $Y \rightarrow S^{1}$, such that with respect to this identification:

- In $N$, parallel transport via the natural connection on $Y \rightarrow S^{1}$ is linear.

- The restriction to $E$ of the almost complex structure $J$ is given by a constant matrix on each fiber of $N \rightarrow S^{1}$.

The normal derivative along $\gamma$ of the identication (31) determines a trivialization $\tau$ of $\left.E\right|_{\gamma}$. With respect to the identification (31) (resp. the trivialization $\tau$ ), the covariant derivative in $S^{1} \times \mathbb{R}^{2}$ near $\gamma$ (resp. $E$ ) is given by

$$
\nabla_{t}=\partial_{t}-J(t) S(t)
$$

Here $t$ denotes the $S^{1}$ coordinate, $J(t)$ is the restriction to $E$ of the almost complex structure, and $S(t)$ is a $2 \times 2$ matrix which is symmetric with respect to the metric determined by $J(t)$ and $\omega$.

Suppose a flow line has an incoming end at $\gamma$ of multiplicity $q$. For $s \ll 0$, the projection of the end to $\mathbb{R} \times S^{1}$ is a covering without branch points. Thus for 
$R \ll 0$, we can describe the end by a pseudoholomorphic map of bundles over $(-\infty, R) \times S^{1}$

$$
u:(-\infty, R) \times \tilde{S^{1}} \longrightarrow(-\infty, R) \times Y
$$

where $\tilde{S}^{1}$ denotes a $q$-fold connected covering of $S^{1}$.

Let $\tilde{t}$ denote the $\tilde{S}^{1}$ coordinate, and let $t$ denote the projection of $\tilde{t}$ to $S^{1}$. Define a self-adjoint operator

$$
A=-J(t) \nabla_{\tilde{t}}=-J(t) \partial_{\tilde{t}}-S(t): C^{\infty}\left(\tilde{S^{1}}, \mathbb{R}^{2}\right) \rightarrow C^{\infty}\left(\tilde{S^{1}}, \mathbb{R}^{2}\right)
$$

By analytic perturbation theory [13], as used in [9], there is a countable set of eigenfunctions $\left\{e_{n}\right\}$ with $A e_{n}=\lambda_{n} e_{n}$, which constitute an orthonormal basis for $L^{2}\left(\tilde{S^{1}}, \mathbb{R}^{2}\right)$ over $\mathbb{R}$.

Lemma 6.3 (asymptotic expansion). If a flow line has an incoming end at $\gamma$ of multiplicity $q$, then we can expand the end for $s \ll 0$ as

$$
u(s, \tilde{t})=\sum_{n} a_{n} e^{\lambda_{n} s} e_{n}(\tilde{t})
$$

with $a_{n} \in \mathbb{R}$. If $a_{n} \neq 0$, then $\lambda_{n}$ is positive.

Proof. For fixed $s$, we can expand $u(s, \cdot)$ as a linear combination of the $e_{n}$ 's. By the admissibility conditions at the beginning of this section, $u$ is pseudoholomorphic near $\gamma$ if and only if it satisfies the linear equation

$$
\left(\partial_{s}-A\right) u(s, \cdot)=0 .
$$

This implies (33). Now we must have $u(s, \tilde{t}) \rightarrow 0$ as $s \rightarrow-\infty$. In particular

$$
a_{n} e^{\lambda_{n} s}=\left\langle u(s, \cdot), e_{n}\right\rangle \rightarrow 0,
$$

which implies that $a_{n}=0$ unless $\lambda_{n}>0$.

If $e$ is a (nonzero) eigenfunction of $A$, then $e(\tilde{t}) \neq 0$ for all $\tilde{t}$, by uniqueness of solutions to ODE's. Thus we can define the winding number $\eta(e) \in \mathbb{Z}$ of the path $e$ in $\mathbb{R}^{2}$ around the origin.

\section{Lemma 6.4 (eigenvalues and winding numbers).}

(a) If $e, e^{\prime}$ are eigenfunctions of $A$ corresponding to eigenvalues $\lambda \leq \lambda^{\prime}$, then $\eta(e) \leq \eta\left(e^{\prime}\right)$.

(b) For each integer $\eta$, the space of eigenfunctions with winding number $\eta$ is 2-dimensional.

(c) Our assumption that $\gamma$ is nondegenerate (so that 0 is not an eigenvalue of A) implies that the maximal winding number for a negative eigenvalue is $\left\lfloor\mu_{\tau}\left(\gamma^{q}\right) / 2\right\rfloor$, and the minimal winding number for a positive eigenvalue is $\left\lceil\mu_{\tau}\left(\gamma^{q}\right) / 2\right\rceil$.

Proof. This is all proved in $[9, \S 3]$. 
Example 6.5. For the simplest kind of elliptic end with monodromy angle $\theta$, if $q=1, J(t)=\left(\begin{array}{cc}0 & -1 \\ 1 & 0\end{array}\right)$, and $J(t) S(t)=\left(\begin{array}{cc}0 & -2 \pi \theta \\ 2 \pi \theta & 0\end{array}\right)$, then identifying $\mathbb{R}^{2}$ with $\mathbb{C}$, we can take $e_{n}(t)=e^{2 \pi i n t}$ for $n \in \mathbb{Z}$, with eigenvalues $\lambda_{n}=2 \pi(n-\theta)$.

Now let $\xi$ be the braid corresponding to an incoming end at $\gamma$ of multiplicity $q$, for $s \ll 0$. We assume that this end is nontrivial, i.e. not a trivial cylinder. We temporarily ignore any other ends of the flow line at $\gamma$. Let $\eta_{\tau}(\xi)$ denote the winding number of $\xi$ around $\gamma$, with respect to the trivialization $\tau$.

Lemma 6.6 (winding bound). The winding number $\eta_{\tau}(\xi)$ is well defined, and

$$
\eta_{\tau}(\xi) \geq\left\lceil\frac{\mu_{\tau}\left(\gamma^{q}\right)}{2}\right\rceil .
$$

Proof. When $s \ll 0$, the smallest eigenvalue $\lambda$ in the expansion (33) dominates, SO

$$
u(s, \tilde{t}) \sim e^{\lambda s} \varphi(\tilde{t})
$$

where $A \varphi=\lambda \varphi$. Thus $\eta_{\tau}(\xi)=\eta(\varphi)$. By Lemma 6.3, $\lambda>0$, so by Lemma 6.4(c), $\eta(\varphi) \geq\left\lceil\mu_{\tau}\left(\gamma^{q}\right) / 2\right\rceil$.

\subsection{The writhe of a single end}

Lemma 6.7. Suppose a flow line has a nontrivial incoming end at $\gamma$ with multiplicity $q$. Let $\xi$ be the corresponding braid, ignoring any other ends at $\gamma$. Then the writhe is bounded by

$$
w_{\tau}(\xi) \geq(q-1) \eta_{\tau}(\xi)
$$

Proof. We will prove inductively that (36) holds for any braid $\xi$ coming from an expansion (33) for $s \ll 0$. Write $\eta=\eta_{\tau}(\xi)$. We begin with the case in which

$$
\operatorname{gcd}(q, \eta)=1 \text {. }
$$

Let $\varphi$ denote the dominating eigenfunction in the expansion (33), as in the proof of Lemma 6.6. Let $\bar{\xi}$ denote the braid swept out by $\varphi$.

Claim: over each point in $S^{1} \times\{0\} \simeq \gamma$, each ray in $\mathbb{R}^{2}$ intersects at most one strand of the braid $\bar{\xi}$; and hence the same is true for $\xi$. To prove the claim, suppose to the contrary that for some $\tilde{t} \in \tilde{S^{1}} \simeq \mathbb{R} / q \mathbb{Z}$ and some $j \in\{1, \ldots, q-1\}$, there is a positive real number $\rho$ with

$$
\varphi(\tilde{t})=\rho \varphi(\tilde{t}+j) .
$$

By uniqueness of solutions to the ODE

$$
\left[-J(t) \partial_{\tilde{t}}-S(t)\right] \varphi(\tilde{t})=\lambda \varphi(\tilde{t}),
$$


we would have

$$
\varphi(\tilde{t}+z)=\rho \varphi(\tilde{t}+j+z)
$$

for all $z \in \tilde{S}^{1}$. Taking $z=j, 2 j, \ldots$, it follows that $\rho=1$ and

$$
\varphi(z)=\varphi(\operatorname{gcd}(j, q)+z)
$$

for all $z \in \tilde{S}^{1}$. This periodicity implies that the winding number $\eta$ is divisible by $q / \operatorname{gcd}(j, q)$. This contradicts our assumption (37), since this divisor also divides $q$ and is larger than 1 . This proves the claim.

From the claim, it follows that $\xi$ is isotopic to a $(q, \eta)$ torus braid, and this braid has writhe $\eta(q-1)$.

Suppose now that

$$
\operatorname{gcd}(q, \eta)=d>1
$$

One can obtain an eigenfunction on $\tilde{S}^{1}$ with winding number $\eta$ by pulling back an eigenfunction on a $(q / d)$-fold covering $\hat{S}^{1}$ of $S^{1}$ with winding number $\eta / d$. By Lemma 6.4(b), this construction yields all eigenfunctions with winding number $\eta$. In particular, the dominating eigenfunction $\varphi_{1}$ in $\xi$ is pulled back from an eigenfunction on $\hat{S}^{1}$, whose braid $\xi_{1}$ has $q / d$ strands and winding number $\eta / d$. It follows that $\xi$ is the cabling of $\xi_{1}$ by some braid $\xi_{2}$ with $d$ strands. That is, $\xi$ is obtained by replacing the string of $\xi_{1}$ with the braid $\xi_{2}$ in a tubular neighborhood of $\xi_{1}$. The braid $\xi_{2}$ is obtained by subtracting the $\varphi_{1}$ term from the expansion of $\xi$, and lifting the resulting braid to a $(q / d)$-fold covering of the tubular neighborhood $N$. Thus $\xi_{2}$ is approximated by an eigenfunction $\varphi_{2}$ with eigenvalue larger than that of $\varphi_{1}$. Let $\eta^{\prime}$ denote the winding number of $\xi_{2}$. Then by Lemma 6.4(a),

$$
\eta^{\prime} \geq \eta
$$

Since $\xi$ is the cabling of $\xi_{1}$ by $\xi_{2}$, it follows from the definition of writhe that

$$
w_{\tau}(\xi)=d^{2} w_{\tau}\left(\xi_{1}\right)+w_{\tau}\left(\xi_{2}\right)
$$

We may inductively assume that

$$
\begin{aligned}
& w_{\tau}\left(\xi_{1}\right) \geq \frac{\eta}{d}\left(\frac{q}{d}-1\right), \\
& w_{\tau}\left(\xi_{2}\right) \geq \eta^{\prime}(d-1) .
\end{aligned}
$$

Putting (40) into (39) and using (38) gives (36).

Lemma 6.8 (writhe bound). Suppose a flow line has a nontrivial incoming end at $\gamma$ with multiplicity $q$ and braid $\xi$, ignoring all other ends. Then

$$
w_{\tau}(\xi) \geq\left\lceil\frac{\mu_{\tau}\left(\gamma^{q}\right)}{2}\right\rceil(q-1) .
$$


If equality holds, then:

(i) If $\gamma$ is hyperbolic with positive eigenvalues then $q=1$.

(ii) If $\gamma$ is hyperbolic with negative eigenvalues, then $q$ is odd or $q=2$.

Proof. Putting Lemma 6.6 into Lemma 6.7, we obtain the inequality (41). Now suppose that equality holds in (41). Since $q>1$, equality must also hold in (35):

$$
\eta=\eta_{\tau}(\xi)=\left\lceil\frac{\mu_{\tau}\left(\gamma^{q}\right)}{2}\right\rceil .
$$

Suppose (i) or (ii) fails; we will obtain a contradiction.

$\underline{\text { Case } 1:} \gamma$ is hyperbolic with positive eigenvalues and $q>1$. By Proposition 2.1 and equation (7), we can choose the trivialization $\tau$ so that $\mu_{\tau}\left(\gamma^{q}\right)=0$. By (42), $\eta=0$. As in the proof of Lemma 6.7, we deduce that $\xi$ is the cabling of a one-strand braid $\xi_{1}$ with winding number zero by a $q$-strand braid $\xi_{2}$ with winding number $\eta^{\prime}$, whose dominant eigenvalue is greater than that of $\xi$. The latter eigenvalue is positive, and by Lemma 6.4(c) there is an eigenfunction with a negative eigenvalue and the same winding number. So by Lemma 6.4(b), $\eta^{\prime} \neq \eta$, and then by Lemma 6.4(a), $\eta^{\prime}>\eta$. By (39) and (40), we deduce that $w_{\tau}(\xi) \geq \eta^{\prime}(q-1)>0$. So equality does not hold in (41), and this is a contradiction.

Case 2: $\gamma$ is hyperbolic with negative eigenvalues, $q$ is even, and $q>2$. By Proposition 2.1 and equation (7), we can choose the trivialization $\tau$ so that $\mu_{\tau}\left(\gamma^{q}\right)=q$. By (42), $\eta=q / 2$. Similarly to Case 1 , we deduce that $\xi$ is the cabling of a 2 -strand braid $\xi_{1}$ by a $q / 2$-strand braid $\xi_{2}$ with winding number $\eta^{\prime}>\eta$. By (39) and (40), and using the fact that $d=q / 2>1$, we obtain $w_{\tau}(\xi)>(q / 2)(q-1)$. So equality does not hold in (41), a contradiction.

\subsection{Linking of two ends}

Let $\xi_{1}$ and $\xi_{2}$ be two disjoint braids in a neighborhood of $\gamma$, and let $\tau \in \mathscr{T}(\gamma)$. We define the linking number

$$
\ell_{\tau}\left(\xi_{1}, \xi_{2}\right) \in \mathbb{Z}
$$

to be one half the signed number of crossings of a strand of $\xi_{1}$ with a strand of $\xi_{2}$, in the projection to $A$ from equation (10), using the same sign convention as for the writhe in $\S 3.1$. The linking number is clearly symmetric: $\ell_{\tau}\left(\xi_{1}, \xi_{2}\right)=\ell_{\tau}\left(\xi_{2}, \xi_{1}\right)$.

Lemma 6.9 (linking bound). Suppose a flow line has a nontrivial incoming end at $\gamma$ of multiplicity $q_{i}$ with braid $\xi_{i}$ and winding number $\eta_{i}$ for $i=1,2$. Then

$$
\ell_{\tau}\left(\xi_{1}, \xi_{2}\right) \geq \min \left(q_{1} \eta_{2}, q_{2} \eta_{1}\right)
$$

Proof. Let $A_{q}$ denote the operator (32) on a $q$-fold cover of $\gamma$. Let $\lambda_{i}$ denote the smallest eigenvalue of $A_{q_{i}}$ in the expansion of $\xi_{i}$. WLOG $\lambda_{1} \leq \lambda_{2}$. We can pull 
back the corresponding eigenfunctions to a $q_{1} q_{2}$-fold cover of $S^{1}$, and applying Lemma 6.4(a) to $A_{q_{1} q_{2}}$, we find that

$$
\eta_{1} q_{2} \leq \eta_{2} q_{1} .
$$

If $\lambda_{1}<\lambda_{2}$, then for $s \ll 0$, equation (33) implies that the braid $\xi_{2}$ is nested inside $\xi_{1}$, i.e. there is a tube containing $\gamma$ and $\xi_{2}$ but not intersecting $\xi_{1}$. It follows from the definition of linking number that

$$
\ell_{\tau}\left(\xi_{1}, \xi_{2}\right)=\eta_{1} q_{2}
$$

Together with (44), this proves (43).

If $\lambda_{1}=\lambda_{2}$, then equation (44) gives $\eta_{1} q_{2}=\eta_{2} q_{1}$. If the coefficients $a$ of the corresponding eigenfunctions in the expansions (33) of $\xi_{1}$ and $\xi_{2}$ are different, then for $s \ll 0$, one can isotope one of the braids radially towards $\gamma$ without intersecting the other, so we obtain (45), which proves (43) as before.

Otherwise, let $\lambda$ be the smallest eigenvalue of $A_{q_{1} q_{2}}$ for which the coefficients of the corresponding eigenfunctions in $\xi_{1}$ and $\xi_{2}$ are different. Let $\xi_{3}$ be the braid obtained from the parts of the expansions of $\xi_{1}$ and $\xi_{2}$ involving eigenvalues smaller than $\lambda$. Let $q_{3}$ be the number of strands of $\xi_{3}$, and let $w_{3}$ denote its writhe in the trivialization $\tau$. Then for $s \ll 0$ and $i=1,2$, the braid $\xi_{i}$ is a cabling of $\xi_{3}$ by some braid $\xi_{i}^{\prime}$ with $q_{i} / q_{3}$ strands, obtained from the remaining terms in the expansion, and we can perform both cablings in the same tubular neighborhood of $\xi_{3}$. It follows from the definition of linking number that

$$
\ell_{\tau}\left(\xi_{1}, \xi_{2}\right)=w_{3} \frac{q_{1}}{q_{3}} \frac{q_{2}}{q_{3}}+\ell_{\tau}\left(\xi_{1}^{\prime}, \xi_{2}^{\prime}\right) .
$$

As in the proof of Lemma 6.7, the braid $\xi_{i}^{\prime}$ has winding number $\eta_{i}^{\prime} \geq \eta_{i}$. By the above reasoning, WLOG,

$$
\ell_{\tau}\left(\xi_{1}^{\prime}, \xi_{2}^{\prime}\right)=\eta_{1}^{\prime} \frac{q_{2}}{q_{3}} \geq \eta_{1} \frac{q_{2}}{q_{3}} .
$$

Now $\xi_{3}$ has winding number

$$
\eta_{3}=\eta_{1} \frac{q_{3}}{q_{1}}=\eta_{2} \frac{q_{3}}{q_{2}}
$$

and the proof of Lemma 6.7 shows that

$$
w_{3} \geq \eta_{3}\left(q_{3}-1\right)=\frac{\eta_{1} q_{3}}{q_{1}}\left(q_{3}-1\right) .
$$

Putting the inequalities (48) and (47) into equation (46), we obtain $\ell_{\tau}\left(\xi_{1}, \xi_{2}\right) \geq$ $\eta_{1} q_{2}$, which proves (43). 


\subsection{A combinatorial lemma}

As we will see in $\S 6.5$, the inequalities we have just established reduce the proof of Proposition 6.1 to the following combinatorial lemma.

Lemma 6.10 (workhorse inequality). Let $\gamma$ be a periodic orbit, let $\tau \in \mathscr{T}(\gamma)$, let $q_{1}, \ldots, q_{k}$ be positive integers, $n=\sum_{i} q_{i}$, and $\rho_{i}=\left\lceil\mu_{\tau}\left(\gamma^{q_{i}}\right) / 2\right\rceil$. Then

$$
\sum_{i=1}^{k}\left(\mu_{\tau}\left(\gamma^{q_{i}}\right)-\rho_{i}\right)+\sum_{i, j=1}^{k} \min \left(q_{i} \rho_{j}, q_{j} \rho_{i}\right) \geq \sum_{i=1}^{n} \mu_{\tau}\left(\gamma^{i}\right) .
$$

Equality holds if and only if:

(i) If $\gamma$ is hyperbolic with negative eigenvalues, then all $q_{i}$ 's are even, except that one $q_{i}$ might equal one.

(ii) If $\gamma$ is elliptic with monodromy angle $\theta$, then

$$
\left\{q_{1}, \ldots, q_{k}\right\}=p_{\text {in }}(\theta, n) .
$$

Proof. We first note that the validity of the inequality (49) does not depend on the choice of trivialization, as changing $\tau$ changes both sides of (49) equally, by adding an integer multiple of $n^{2}+n$. We now consider three cases.

Case 1: $\gamma$ is hyperbolic with positive eigenvalues. We can choose the trivialization $\tau$ so that $\mu_{\tau}\left(\gamma^{i}\right)=0$ for all $i$. Then the inequality (49) trivially holds, and is an equality, as all terms in it are equal to zero.

Case 2: $\gamma$ is elliptic with monodromy angle $\theta$ with respect to the trivialization $\tau$. Then $\rho_{i}=\left\lceil q_{i} \theta\right\rceil$. Define

$$
M_{\theta}\left(q_{1}, \ldots, q_{k}\right)=\sum_{i=1}^{k}\left\lfloor q_{i} \theta\right\rfloor+\sum_{i, j=1}^{k} \min \left(q_{i}\left\lceil q_{j} \theta\right\rceil, q_{j}\left\lceil q_{i} \theta\right\rceil\right)-n-2 \sum_{i=1}^{n}\lfloor i \theta\rfloor .
$$

Lemma 6.10 reduces in this case to the following lemma. This characterization of the incoming partition will also be useful in $§ 7$.

Lemma 6.11 (incoming partitions). If $\theta$ is an irrational number, and $q_{1}, \ldots, q_{k}$ are positive integers whose sum is $n$, then:

(a) $M_{\theta}\left(q_{1}, \ldots, q_{k}\right) \geq 0$.

(b) Equality holds in (a) if and only if $\left\{q_{1}, \ldots, q_{k}\right\}=p_{\text {in }}(\theta, n)$.

Proof. Recall the notation $f_{\theta}(q)=q^{-1}\lceil q \theta\rceil$ from $\$ 4$.1. By symmetry, we may assume that

$$
f_{\theta}\left(q_{1}\right) \geq f_{\theta}\left(q_{2}\right) \geq \cdots \geq f_{\theta}\left(q_{k}\right) .
$$

(a) By induction, it will suffice to show that $M_{\theta}\left(q_{1}, \ldots, q_{k}\right) \geq M_{\theta}\left(q_{1}, \ldots, q_{k-1}\right)$. Under the ordering convention (50), the latter inequality becomes

$$
\left\lfloor q_{k} \theta\right\rfloor+q_{k}\left\lceil q_{k} \theta\right\rceil+2 n^{\prime}\left\lceil q_{k} \theta\right\rceil \geq q_{k}+2 \sum_{i=n^{\prime}+1}^{n}\lfloor i \theta\rfloor
$$


where $n^{\prime}=q_{1}+\cdots+q_{k-1}$. Since $\theta$ is irrational, we can convert floors to ceilings to rewrite this as $z(\theta) \geq 0$, where

$$
z(\xi)=\left(2 n^{\prime}+q_{k}+1\right)\left\lceil q_{k} \xi\right\rceil+q_{k}-1-2 \sum_{i=n^{\prime}+1}^{n}\lceil i \xi\rceil .
$$

Let $\theta^{\prime}=f_{\theta}\left(q_{k}\right)$. We observe that

$$
z(\theta) \geq z\left(\theta^{\prime}\right),
$$

because as a function of $\xi \in\left[\theta, \theta^{\prime}\right]$, the first term on the right side of (51) is constant, while the remaining terms are monotone decreasing. So to prove (a), it will suffice to show that

$$
z\left(\theta^{\prime}\right) \geq 0
$$

Let $\epsilon_{i}(\xi)=\lceil i \xi\rceil-i \xi$. Then (51) can be rewritten as

$$
z(\xi)=\left(2 n^{\prime}+q_{k}+1\right) \epsilon_{q_{k}}(\xi)+q_{k}-1-2 \sum_{i=n^{\prime}+1}^{n} \epsilon_{i}(\xi) .
$$

The numbers $\epsilon_{n^{\prime}+1}\left(\theta^{\prime}\right), \ldots, \epsilon_{n}\left(\theta^{\prime}\right)$ are evenly spaced around the circle $\mathbb{R} / \mathbb{Z}$ with spacing $1 / s$, where $s=q_{k} / \operatorname{gcd}\left(\left\lceil q_{k} \theta\right\rceil, q_{k}\right)$. So

$$
\begin{aligned}
\sum_{i=n^{\prime}+1}^{n} \epsilon_{i}\left(\theta^{\prime}\right) & =\frac{q_{k}}{s}\left(\frac{1}{s}+\frac{2}{s}+\cdots+\frac{s-1}{s}\right) \\
& =\frac{q_{k}}{2}\left(\frac{s-1}{s}\right) \\
& \leq \frac{q_{k}-1}{2} .
\end{aligned}
$$

Putting this inequality into equation (54) with $\xi=\theta^{\prime}$, and noting that $\epsilon_{q_{k}}\left(\theta^{\prime}\right)=0$, we obtain the desired inequality (53), so (a) is proved.

(b) We have $M_{\theta}\left(q_{1}, \ldots, q_{k}\right)=0$ if and only if equality holds in the inequalities (52) and (55) at each stage in the above induction. Now equality holds in (52) at each stage if and only if

(i) Under the ordering convention (50), the interval $\left(\theta, f_{\theta}\left(q_{i}\right)\right)$ contains no rational numbers of the form $p / r$ with

$$
q_{1}+\cdots+q_{i-1}<r \leq q_{1}+\cdots+q_{i} .
$$

And equality holds in (55) at each stage if and only if

(ii) $\operatorname{gcd}\left(q_{i},\left\lceil q_{i} \theta\right\rceil\right)=1$.

For a given integer $r$, there exists an integer $p$ with $p / r \in\left(\theta, f_{\theta}\left(q_{i}\right)\right)$ if and only if $f_{\theta}(r)<f_{\theta}\left(q_{i}\right)$. Thus condition (i) is equivalent to

(i') $f_{\theta}\left(q_{i}\right) \leq f_{\theta}(r)$ for all $r$ in the range (56). 
Also, condition (ii) is equivalent to

(ii') $f_{\theta}\left(q_{i}\right) \neq f_{\theta}(r)$ for all $r<q_{i}$.

This is because if $f_{\theta}\left(q_{i}\right)=f_{\theta}(r)$ with $r<q_{i}$, i.e. $\left\lceil q_{i} \theta\right\rceil / q_{i}=\lceil r \theta\rceil / r$, then $q_{i}$ and $\left\lceil q_{i} \theta\right\rceil$ have a common factor; while conversely, if $q_{i}$ and $\left\lceil q_{i} \theta\right\rceil$ are both divisible by $d$, then $f_{\theta}\left(q_{i}\right)=f_{\theta}\left(q_{i} / d\right)$.

Now suppose that conditions (i') and (ii') hold. By (i') and the ordering convention (50), $f_{\theta}\left(q_{i}\right) \leq f_{\theta}(r)$ for all $r \in\left[1, q_{1}+\cdots+q_{i}\right]$. Together with (ii'), this implies that

$$
q_{i}=\max \left(S_{\theta} \cap\left[1, q_{1}+\cdots+q_{i}\right]\right) .
$$

Taking $i=k, k-1, \ldots$, we deduce inductively that $\left\{q_{1}, \ldots, q_{k}\right\}=p_{\text {in }}(\theta, n)$. Conversely, if $\left\{q_{1}, \ldots, q_{k}\right\}=p_{\text {in }}(\theta, n)$, then by the ordering (50), equation (57) holds by the definition of $p_{\text {in }}(\theta, n)$, and this implies (i') and (ii').

This completes the proof of Lemma 6.11.

$\underline{\text { Case } 3}$ of the proof of Lemma 6.10: $\gamma$ is hyperbolic with negative eigenvalues. We can choose the trivialization $\tau$ so that $\mu_{\tau}\left(\gamma^{q}\right)=q$ and $\rho_{i}=\left\lceil q_{i} / 2\right\rceil$. We then need to show that $M_{1 / 2}\left(q_{1}, \ldots, q_{k}\right) \geq 0$, where

$$
M_{1 / 2}\left(q_{1}, \ldots, q_{k}\right)=-\sum_{i=1}^{k}\left\lceil\frac{q_{i}}{2}\right\rceil+\sum_{i, j=1}^{k} \min \left(q_{i}\left\lceil\frac{q_{j}}{2}\right\rceil, q_{j}\left\lceil\frac{q_{i}}{2}\right\rceil\right)-\frac{n(n-1)}{2}
$$

with equality if and only if all $q_{i}$ 's are even, except that one $q_{i}$ might equal 1 . The proof parallels the proof of Lemma 6.11, but is simpler. Without loss of generality,

$$
q_{1}^{-1}\left\lceil\frac{q_{1}}{2}\right\rceil \geq q_{2}^{-1}\left\lceil\frac{q_{2}}{2}\right\rceil \geq \cdots \geq q_{k}^{-1}\left\lceil\frac{q_{k}}{2}\right\rceil .
$$

We then calculate that

$$
M_{1 / 2}\left(q_{1}, \ldots, q_{k}\right)-M_{1 / 2}\left(q_{1}, \ldots, q_{k-1}\right)=\left(\left\lceil\frac{q_{k}}{2}\right\rceil-\frac{q_{k}}{2}\right)\left(2 n-q_{k}-1\right) .
$$

This is nonnegative since $1 \leq q_{k} \leq n$, and zero if and only if $q_{k}$ is even or $2 n-q_{k}-1=0$, i.e. $n=q_{k}=1$. We are done by induction on $k$.

This completes the proof of Lemma 6.10.

\subsection{Flow lines without trivial cylinders}

We now put everything together to finish the proof of Proposition 6.1. Let $C$ be a flow line without trivial cylinders from $\left\{\left(\alpha_{i}, m_{i}\right)\right\}$ to $\left\{\left(\beta_{j}, n_{j}\right)\right\}$. We start with a local inequality for the incoming ends. Recall that for each $j$, the incoming ends of $C$ at $\beta_{j}$ determine a partition $p_{j}^{-}=\left(q_{j, 1}^{\prime}, q_{j, 2}^{\prime}, \ldots\right)$ of $n_{j}$, and a braid $\xi_{j}^{-}$in a neighborhood of $\beta_{j}$ with $n_{j}$ strands. 
Lemma 6.12 (incoming inequality). For a fixed $j$, write $\gamma=\beta_{j}, n=n_{j}, \xi=\xi_{j}^{-}$, and $q_{r}=q_{j, r}^{\prime}$. Let $\tau \in \mathscr{T}(\gamma)$. Then

$$
w_{\tau}(\xi)+\sum_{r} \mu_{\tau}\left(\gamma^{q_{r}}\right) \geq \sum_{i=1}^{n} \mu_{\tau}\left(\gamma^{i}\right) .
$$

Equality holds only if $\left\{q_{1}, q_{2}, \ldots\right\}=p_{\text {in }}(\gamma, n)$.

Proof. The braid $\xi$ has components $\xi_{1}, \ldots, \xi_{k}$, where $\xi_{i}$ has $q_{i}$ strands and winding number $\eta_{i}$. Let $\rho_{i}=\left\lceil\mu_{\tau}\left(\gamma^{q_{i}}\right) / 2\right\rceil$. By Lemma 6.6, $\eta_{i} \geq \rho_{i}$. Then

$$
\begin{aligned}
w_{\tau}(\xi) & =\sum_{i=1}^{k} w_{\tau}\left(\xi_{i}\right)+\sum_{i \neq j} \ell_{\tau}\left(\xi_{i}, \xi_{j}\right) \\
& \geq \sum_{i=1}^{k} \rho_{i}\left(q_{i}-1\right)+\sum_{i \neq j} \min \left(q_{i} \rho_{j}, q_{j} \rho_{i}\right) .
\end{aligned}
$$

The equality in the first line follows from the definitions of writhe and linking number. The second line follows from Lemmas 6.8 and 6.9. Combining this with Lemma 6.10, we obtain the desired inequality (58).

If equality holds in (58), then conditions 6.8(i) and (ii) hold for each $q_{i}$, since we applied Lemma 6.8 to each $\xi_{i}$, and conditions 6.10(i) and (ii) hold. All together, these conditions imply that $\left\{q_{1}, q_{2}, \ldots\right\}=p_{\text {in }}(\gamma, n)$.

By a symmetry argument, we can obtain a similar local inequality for the outgoing ends. If $C$ is a flow line as above, then for each $i$, the outgoing ends of $C$ at $\alpha_{i}$ determine a partition $p_{i}^{+}=\left(q_{i, 1}, q_{i, 2}, \ldots\right)$ of $m_{i}$, and a braid $\xi_{i}^{+}$in a neighborhood of $\alpha_{i}$ with $m_{i}$ strands.

Lemma 6.13 (outgoing inequality). For a fixed $i$, write $\gamma=\alpha_{i}, m=m_{i}, \xi=\xi_{i}^{+}$, and $q_{r}=q_{i, r}$. Let $\tau \in \mathscr{T}(\gamma)$. Then

$$
w_{\tau}(\xi)+\sum_{r} \mu_{\tau}\left(\gamma^{q_{r}}\right) \leq \sum_{l=1}^{m} \mu_{\tau}\left(\gamma^{l}\right)
$$

Equality holds only if $\left\{q_{1}, q_{2}, \ldots\right\}=p_{\text {out }}(\gamma, m)$.

Proof. Let $Y^{\prime}$ denote the mapping torus of $\phi^{-1}$. By equation (1), the map

$$
\begin{aligned}
\mathbb{R} \times(\Sigma \times \mathbb{R}) & \rightarrow \mathbb{R} \times(\Sigma \times \mathbb{R}), \\
(s,(x, t)) & \mapsto(-s,(x,-t))
\end{aligned}
$$

descends to a bijection

$$
\mathbb{R} \times Y \rightarrow \mathbb{R} \times Y^{\prime}
$$

This map sends the almost complex structure on $\mathbb{R} \times Y$ to an admissible almost complex structure on $\mathbb{R} \times Y^{\prime}$, see $\S 1.2$. For this almost complex structure on $\mathbb{R} \times Y^{\prime}$, 
the bijection (61) sends flow lines to flow lines. Since the bijection (61) switches $s$ with $-s$, incoming ends become outgoing ends and vice versa. If $\gamma$ is a periodic orbit in $Y$, let $\gamma^{\prime}$ denote the corresponding periodic orbit in $Y^{\prime}$. Then $C$ can be regarded as a flow line in $\mathbb{R} \times Y^{\prime}$ from $\left\{\left(\beta_{j}^{\prime}, n_{j}\right)\right\}$ to $\left\{\left(\alpha_{i}^{\prime}, m_{i}\right)\right\}$. By Lemma 6.12, the braid $\xi^{\prime}$ at $\alpha_{i}^{\prime}$ satisfies

$$
w_{\tau}\left(\xi^{\prime}\right)+\sum_{r} \mu_{\tau}\left(\gamma^{\prime q_{r}}\right) \geq \sum_{l=1}^{m} \mu_{\tau}\left(\gamma^{\prime l}\right),
$$

in the notation above equation (60). Because the bijection (61) switches $t$ with $-t$, we have $w_{\tau}\left(\xi^{\prime}\right)=-w_{\tau}(\xi)$ and $\mu_{\tau}\left(\gamma^{\prime l}\right)=-\mu_{\tau}\left(\gamma^{l}\right)$. Thus we obtain the inequality (60).

If equality holds in (60), then since we used Lemma 6.12, we must have $\left\{q_{1}, \ldots, q_{k}\right\}=p_{\text {in }}\left(\gamma^{\prime}, m\right)$. But $p_{\text {in }}\left(\gamma^{\prime}, m\right)=p_{\text {out }}(\gamma, m)$; because if $\gamma$ is hyperbolic with positive or negative eigenvalues, then so is $\gamma^{\prime}$; and if $\gamma$ is elliptic with monodromy angle $\theta$, then $\gamma^{\prime}$ is elliptic with monodromy angle $-\theta$.

Proposition 6.1 follows immediately from Lemmas 6.12 and 6.13. This proves the Index theorem 1.7 for flow lines without trivial cylinders.

\section{Trivial cylinders}

To complete the proof of the Index theorem 1.7, we need to consider a flow line $C$ which may contain trivial cylinders. We begin with some notation, which will also be used in $\S 8$. If $C$ is a flow line from $\alpha$ to $\beta$, we write $I(C)=I(\alpha, \beta ;[C])$. More generally, if $C_{p}$ is a flow line from the orbit set $\alpha_{p}$ to the orbit set $\beta_{p}$, for $p=1, \ldots, r$, we write

$$
I\left(\sum_{p=1}^{r} C_{p}\right)=I\left(\sum_{p=1}^{r} \alpha_{p}, \sum_{p=1}^{r} \beta_{p} ; \sum_{p=1}^{r}\left[C_{p}\right]\right) .
$$

Here addition of orbit sets is defined by adding the multiplicities of all periodic orbits involved.

Now to prove Theorem 1.7, write $C=C^{\prime} \cup T$, where $T$ is a union of trivial cylinders (possibly repeated), and $C^{\prime}$ contains no trivial cylinders, as in $\S 4.2$. We have $\operatorname{dim}\left(\mathscr{M}_{T}\right)=0$; in fact $\mathscr{M}_{T}=\{T\}$, by Proposition 9.1. Therefore

$$
\operatorname{dim}\left(\mathscr{M}_{C}\right)=\operatorname{dim}\left(\mathscr{M}_{C^{\prime}}\right)
$$

By Lemma 5.2 and Proposition 6.1,

$$
\operatorname{dim}\left(\mathscr{M}_{C^{\prime}}\right) \leq I\left(C^{\prime}\right)
$$

So the following proposition will complete the proof of Theorem 1.7. 
Proposition 7.1 (trivial cylinders). If $C^{\prime}$ is a flow line without trivial cylinders, and if $T$ is a union of trivial cylinders (possibly repeated), then

$$
I\left(C^{\prime}\right) \leq I\left(C^{\prime}+T\right)-2 \#\left(C^{\prime} \cap T\right) .
$$

Equality holds only if $C=C^{\prime} \cup T$ satisfies the admissibility conditions (23) and (24).

Remark 7.2. Of course, if $C$ is a flow line, then by definition $C^{\prime} \cap T=\emptyset$. In general, by intersection positivity [14], the algebraic intersection number $\#\left(C^{\prime} \cap T\right)>0$ whenever $C^{\prime} \cap T \neq \emptyset$.

Lemma 7.3 (split partitions). Let $\theta \in \mathbb{R} \backslash \mathbb{Q}$, and suppose

$$
\lfloor i \theta\rfloor+\lceil m \theta\rceil=\lfloor(m+i) \theta\rfloor
$$

for $i=1, \ldots, n$. Then

$$
p_{\text {in }}(\theta, m+n)=p_{\text {in }}(m, \theta) \cup p_{\text {in }}(\theta, n) .
$$

Proof. Write $p_{\text {in }}(\theta, m)=\left\{q_{1}, \ldots, q_{k}\right\}$ and $p_{\text {in }}(\theta, n)=\left\{r_{1}, \ldots, r_{l}\right\}$. By Lemma 6.11, we have $M_{\theta}\left(q_{1}, \ldots, q_{k}\right)=M_{\theta}\left(r_{1}, \ldots, r_{l}\right)=0$. Using this, we compute that

$$
\begin{aligned}
M_{\theta}\left(q_{1}, \ldots, q_{k}, r_{1}, \ldots, r_{l}\right)= & 2 \sum_{i=1}^{k} \sum_{j=1}^{l} \min \left(q_{i}\left\lceil r_{j} \theta\right\rceil, r_{j}\left\lceil q_{i} \theta\right\rceil\right) \\
& +2 \sum_{i=1}^{n}(\lfloor i \theta\rfloor-\lfloor(m+i) \theta\rfloor) .
\end{aligned}
$$

Using Lemma 4.5(a), we estimate

$$
\sum_{i=1}^{k} \sum_{j=1}^{l} \min \left(q_{i}\left\lceil r_{j} \theta\right\rceil, r_{j}\left\lceil q_{i} \theta\right\rceil\right) \leq \sum_{i=1}^{k} \sum_{j=1}^{l} r_{j}\left\lceil q_{i} \theta\right\rceil=n\lceil m \theta\rceil .
$$

Putting this into the previous equation and then using (63), we obtain

$$
M_{\theta}\left(q_{1}, \ldots, q_{k}, r_{1}, \ldots, r_{l}\right) \leq 2 \sum_{i=1}^{n}(\lfloor i \theta\rfloor-\lfloor(m+i) \theta\rfloor+\lceil m \theta\rceil)=0 .
$$

By Lemma 6.11, it follows that $\left\{q_{1}, \ldots, q_{k}, r_{1}, \ldots, r_{l}\right\}=p_{\text {in }}(\theta, m+n)$.

Proof of Proposition 7.1. Suppose $T$ consists of trivial cylinders over periodic orbits $\gamma_{l}$ repeated $n_{l}$ times. At $\gamma_{l}$, suppose that $C^{\prime}$ has outgoing ends of total multiplicity $m_{l}^{+}$, and incoming ends of total multiplicity $m_{l}^{-}$. (Comparing with the notation of $\S 4.2$, if $\gamma_{l}=\alpha_{i}=\beta_{j}$, then $m_{l}^{+}=m_{i}^{\prime}, m_{l}^{-}=n_{j}^{\prime}$, and $n_{l}=m_{i}-m_{i}^{\prime}=n_{j}-n_{j}^{\prime}$.) Let $\xi_{l}^{+}$and $\xi_{l}^{-}$denote the corresponding braids from $C^{\prime}$. Let $\tau$ be a trivialization of $E$ over the ends of $C$. 
Starting from $C$, we can perturb the trivial cylinders to obtain a surface $S$ which is a representative of $[C]$ as in Definition 2.2 , except for finitely many self-intersections coming from the intersections of $C^{\prime}$ with $T$. We have

$$
c_{\tau}(S)=c_{\tau}\left(C^{\prime}\right)
$$

because the trivializations of $E$ are the same over both ends of each cylinder. As in the relative adjunction formula (18),

$$
\begin{aligned}
Q_{\tau}(S)-Q_{\tau}\left(C^{\prime}\right)-2 \#\left(C^{\prime} \cap T\right) & =w_{\tau}\left(C^{\prime}\right)-w_{\tau}(S) \\
& =2 \sum_{l} n_{l}\left(\eta_{\tau}\left(\xi_{l}^{-}\right)-\eta_{\tau}\left(\xi_{l}^{+}\right)\right) .
\end{aligned}
$$

And by definition of $\mu_{\tau}$, we have

$$
\mu_{\tau}(S)-\mu_{\tau}\left(C^{\prime}\right)=\sum_{l} \sum_{k=1}^{n_{l}}\left(\mu_{\tau}\left(\gamma_{l}^{m_{l}^{+}+k}\right)-\mu_{\tau}\left(\gamma_{l}^{m_{l}^{-}+k}\right)\right) .
$$

By the above three equations, in order to prove Proposition 7.1, it will suffice to show that for each $l$ and $1 \leq k \leq n_{l}$,

$$
2\left(\eta_{\tau}\left(\xi_{l}^{+}\right)-\eta_{\tau}\left(\xi_{l}^{-}\right)\right) \leq \mu_{\tau}\left(\gamma_{l}^{m_{l}^{+}+k}\right)-\mu_{\tau}\left(\gamma_{l}^{m_{l}^{-}+k}\right)
$$

with equality for all such $k$ only if $C$ satisfies (23) and (24). In the rest of this proof, we drop the subscripts ' $l$ ' and ' $\tau$ '.

Let $\left(q_{1}^{+}, q_{2}^{+}, \ldots\right)$ and $\left(q_{1}^{-}, q_{2}^{-}, \ldots\right)$ denote the partitions of $m^{+}$and $m^{-}$determined by the ends of $C^{\prime}$ at $\gamma$. Let $\rho_{r}^{+}=\left\lfloor\mu\left(\gamma^{q_{r}^{+}}\right) / 2\right\rfloor$ and $\rho_{s}^{-}=\left\lceil\mu\left(\gamma^{q_{s}^{-}}\right) / 2\right\rceil$. By Lemma 6.6, we have $\eta\left(\xi^{+}\right) \leq \sum_{r} \rho_{r}^{+}$and $\eta\left(\xi_{i}\right) \geq \sum_{s} \rho_{s}^{-}$. (We know this even without assuming $(\phi, J)$ is admissible at $\gamma$, by the asymptotics in [9].) So it will suffice to show that for $1 \leq k \leq n$,

$$
2\left(\sum_{r} \rho_{r}^{+}-\sum_{s} \rho_{s}^{-}\right) \leq \mu\left(\gamma^{m^{+}+k}\right)-\mu\left(\gamma^{m^{-}+k}\right),
$$

with equality for all such $k$ only if (23) and (24) hold for $C$ at $\gamma$. To prove this, we consider three cases.

Case 1: $\gamma$ is hyperbolic with positive eigenvalues. We can choose the trivialization $\bar{\tau}$ so that $\mu\left(\gamma^{k}\right)=0$ for all $k$. Then all terms in (64) are zero, so (64) holds. The admissibility conditions (23) and (24) are automatically satisfied in this case.

Case 2: $\gamma$ is hyperbolic with negative eigenvalues. We can choose the trivialization so that $\mu\left(\gamma^{k}\right)=k$ for all $k$. The inequality (64) asserts in this case that

$$
2\left(\sum_{r}\left\lfloor\frac{q_{r}^{+}}{2}\right\rfloor-\sum_{s}\left\lceil\frac{q_{s}^{-}}{2}\right\rceil\right) \leq m^{+}-m^{-} .
$$

This clearly holds. Equality holds only if $\mathrm{m}^{+}$and $\mathrm{m}^{-}$are even, which is the content of (23) and (24) in this case, by Remark 4.8, since $n>0$. 


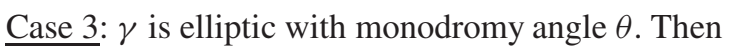

$$
\sum_{r} \rho_{r}^{+}-\sum_{s} \rho_{s}^{-}=\sum_{r}\left\lfloor q_{r}^{+} \theta\right\rfloor-\sum_{s}\left\lceil q_{s}^{-} \theta\right\rceil \leq\left\lfloor m^{+} \theta\right\rfloor+\left\lfloor-m^{-} \theta\right\rfloor,
$$

and

$$
\mu\left(\gamma^{m^{+}+k}\right)-\mu\left(\gamma^{m^{-}+k}\right)=2\left(\left\lfloor\left(m^{+}+k\right) \theta\right\rfloor-\left\lfloor\left(m^{-}+k\right) \theta\right\rfloor\right) .
$$

So in this case (64) follows from

$$
\left\lfloor m^{+} \theta\right\rfloor+\left\lfloor-m^{-} \theta\right\rfloor \leq\left\lfloor\left(m^{+}-m^{-}\right) \theta\right\rfloor \leq\left\lfloor\left(m^{+}+k\right) \theta\right\rfloor-\left\lfloor\left(m^{-}+k\right) \theta\right\rfloor .
$$

Now suppose that equality holds in (64), so that it holds in (65), for $k=$ $1, \ldots, n$. Adding $\lfloor k \theta\rfloor$ to both sides and manipulating, we obtain

$$
\left\lfloor m^{+} \theta\right\rfloor+\lfloor k \theta\rfloor-\left\lfloor\left(m^{+}+k\right) \theta\right\rfloor=\left\lceil m^{-} \theta\right\rceil+\lfloor k \theta\rfloor-\left\lfloor\left(m^{-}+k\right) \theta\right\rfloor .
$$

The left side of this equation cannot be positive, and the right side cannot be negative. Hence both sides equal zero. Since the right side equals zero for $k=$ $1, \ldots, n$, applying Lemma 7.3 gives

$$
p_{\text {in }}\left(\theta, m^{-}+k\right)=p_{\text {in }}\left(\theta, m^{-}\right) \cup p_{\text {in }}(\theta, k) .
$$

This is the admissibility condition (24) for $C$ at $\gamma$. By symmetry, as in the proof of Lemma 6.13, we also obtain the admissibility condition (23).

This completes the proof of Proposition 7.1 and the Index theorem 1.7.

\section{Multiply covered pseudoholomorphic curves}

Having proved the index theorem, we now want to establish compactness results for moduli spaces of flow lines of dimension one and two. A major step is to show roughly that in these moduli spaces, a sequence of flow lines cannot converge to a multiply covered pseudoholomorphic curve, except that there can be repeated trivial cylinders. We will do that in $\$ 9$, by showing that an embedded (or quasiembedded) curve underlying such a multiply covered curve would live in a moduli space of negative expected dimension, and hence does not exist for generic $J$. The key is the following index inequality; see the beginning of $\$ 7$ for the notation.

\subsection{A generalization of the index inequality}

Theorem 8.1 (multiply covered curves). Let $C_{1}, \ldots, C_{r}$ be disjoint flow lines and $d_{1}, \ldots, d_{r}$ positive integers. Assume that $(\phi, J)$ is $d$-admissible, where $d$ is the maximum period of the orbits at the ends of the $C_{p}$ 's, and $J$ is generic. Then

$$
\sum_{p=1}^{r} d_{p} \operatorname{dim}\left(\mathscr{M}_{C_{p}}\right) \leq I\left(\sum_{p=1}^{r} d_{p} C_{p}\right) .
$$


Remark 8.2. Of course this theorem is a generalization of the index inequality (3), which is recovered when $r=d_{1}=1$. We proved the latter separately in order to simplify the exposition.

Remark 8.3. More generally, one might try to show that if $\alpha_{p}$ and $\beta_{p}$ are orbit sets for $p=1, \ldots, r$, and if $Z_{p} \in H_{2}\left(Y ; \alpha_{p}, \beta_{p}\right)$, then

$$
\sum_{p=1}^{r} d_{p} I\left(\alpha_{p}, \beta_{p} ; Z_{p}\right) \leq I\left(\sum_{p=1}^{r} d_{p} \alpha_{p}, \sum_{p=1}^{r} d_{p} \beta_{p} ; \sum_{p=1}^{r} d_{p} Z_{p}\right) .
$$

This, together with (3), would imply Theorem 8.1. However, (67) is not always true; one can use Proposition 1.6(d) to create a counterexample.

\subsection{Topological preliminaries}

Before proving Theorem 8.1, we need some more information about the relative intersection pairing. If $C$ is a flow line without trivial cylinders and $\tau$ is a trivialization of the periodic orbits at the ends, define the total winding number

$$
\eta_{\tau}(C)=\sum_{i} \eta_{\tau}\left(\xi_{i}^{+}\right)-\sum_{j} \eta_{\tau}\left(\xi_{j}^{-}\right)
$$

Here $\xi_{i}^{+}$and $\xi_{j}^{-}$are the braids determined by the outgoing and incoming ends, as in $\S 3$.

The following inequality will be needed in the proof of Theorem 8.1 , and is also useful for showing that flow lines cannot exist under certain conditions. For use in $\$ 9$, instead of just considering flow lines, we will more generally consider quasi-embedded GFL's, see $\$ 9.3$.

Proposition 8.4 (s-translation inequality). If C is a quasi-embedded GFL without trivial cylinders such that $(\phi, J)$ is admissible near its ends, then

$$
Q_{\tau}(C) \geq-w_{\tau}(C)-\eta_{\tau}(C)+2 \delta(C) .
$$

Proof. Let $C^{\prime}$ be the surface in $\mathbb{R} \times Y$ obtained by translating $C$ in the $s$ direction, i.e. the $\mathbb{R}$ direction, by a small positive amount. Since the almost complex structure $J$ is $\mathbb{R}$-invariant, $C^{\prime}$ is pseudoholomorphic. Let $C^{\prime \prime}=C \cup C^{\prime}$. Since $C$ contains no trivial cylinders, $C^{\prime \prime}$ has no multiply covered components, so $C^{\prime \prime}$ is also a quasi-embedded GFL, see $\$ 9.3$. We then have:

$$
\begin{aligned}
c_{\tau}\left(C^{\prime \prime}\right) & =2 c_{\tau}(C), \\
Q_{\tau}\left(C^{\prime \prime}\right) & =4 Q_{\tau}(C), \\
\delta\left(C^{\prime \prime}\right) & =4 \delta(C)+\#\left(C \cap C^{\prime}\right), \\
w_{\tau}\left(C^{\prime \prime}\right) & =4 w_{\tau}(C)+2 \eta_{\tau}(C) .
\end{aligned}
$$

The first three equations follow directly from the definitions. To prove the fourth equation, we observe from the asymptotics in $\$ 6$ that the braid from the outgoing 
(resp. incoming) ends of $C^{\prime}$ at $\gamma$ is obtained by moving the braid from $C$ outward from (resp. inward toward) $\gamma$. Hence the braid for $C^{\prime \prime}$ is the cabling of the braid from $C$ by a two-strand braid whose writhe is twice the winding number. Thus the fourth equation follows from (39).

We now apply equation (18) to $C^{\prime \prime}$, subtract equation (18) applied to $C$ and $C^{\prime}$, and use the above four equations, to obtain

$$
Q_{\tau}(C)+w_{\tau}(C)+\eta_{\tau}(C)-2 \delta(C)=\#\left(C \cap C^{\prime}\right) .
$$

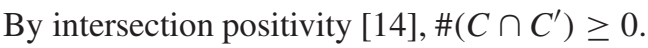

Next, we introduce an extension of the relative intersection pairing $Q$. If $\alpha_{p}$ and $\beta_{p}$ are orbit sets for $p=1,2$, and if $\tau$ is a trivialization of $E$ over all the periodic orbits in $\alpha_{1}, \alpha_{2}, \beta_{1}, \beta_{2}$, then we can define

$$
Q_{\tau}: H_{2}\left(Y ; \alpha_{1}, \beta_{1}\right) \times H_{2}\left(Y ; \alpha_{2}, \beta_{2}\right) \rightarrow \mathbb{Z}
$$

just as in Definition 2.4. Equivalently, if $Z_{p} \in H_{2}\left(Y ; \alpha_{p}, \beta_{p}\right)$ for $p=1,2$, then

$$
Q_{\tau}\left(Z_{1}+Z_{2}, Z_{1}+Z_{2}\right)=Q_{\tau}\left(Z_{1}, Z_{1}\right)+Q_{\tau}\left(Z_{2}, Z_{2}\right)+2 Q_{\tau}\left(Z_{1}, Z_{2}\right) .
$$

For this extended intersection pairing, there is a version of the adjunction formula (13). Let $S_{1}, S_{2}$ be representatives of $Z_{1}, Z_{2}$ which intersect in finitely many points. Let $\left\{\alpha_{i}\right\}$ (resp. $\left.\left\{\beta_{j}\right\}\right)$ denote the set of all periodic orbits at which $S_{1}$ or $S_{2}$ has outgoing (resp. incoming) ends. For $p=1,2$, let $\xi_{p, i}^{+}$(resp. $\xi_{p, j}^{-}$) denote the braid corresponding to the outgoing (resp. incoming) ends of $S_{p}$ at $\alpha_{i}$ (resp. $\beta_{j}$ ). Define the total linking number

$$
\ell_{\tau}\left(S_{1}, S_{2}\right)=\sum_{i} \ell_{\tau}\left(\xi_{1, i}^{+}, \xi_{2, i}^{+}\right)-\sum_{j} \ell_{\tau}\left(\xi_{1, j}^{-}, \xi_{2, j}^{-}\right) .
$$

This is symmetric in $S_{1}$ and $S_{2}$. We then have:

Lemma 8.5. Under the above assumptions,

$$
Q_{\tau}\left(Z_{1}, Z_{2}\right)=-\ell_{\tau}\left(S_{1}, S_{2}\right)+\#\left(S_{1} \cap S_{2}\right) .
$$

Proof. We can create $\tau$-representatives of $Z_{1}$ and $Z_{2}$, whose projected conormals lie in distinct rays, by attaching cobordisms of braids to $S_{1}$ and $S_{2}$ as in the proof of Proposition 3.1(b). The intersection number of these cobordisms equals minus the linking number.

\subsection{Proof of Theorem 8.1}

We begin by assuming that the $C_{p}$ 's do not contain trivial cylinders.

Let $\left\{\alpha_{i}\right\}$ (resp. $\left\{\beta_{j}\right\}$ ) denote the set of all periodic orbits at which any of the $C_{p}$ 's have outgoing (resp. incoming) ends. Choose some trivialization $\tau$ of $E$ over the $\alpha_{i}$ 's and $\beta_{j}$ 's. Let $m_{p, i}$ (resp. $n_{p, j}$ ) denote the total multiplicity of the outgoing (resp. incoming) ends of $C_{p}$ at $\alpha_{i}$ (resp. $\beta_{j}$ ). Let $M_{i}=\sum_{p} d_{p} m_{p, i}$ and 
$N_{j}=\sum_{p} d_{p} n_{p, j}$. Write $\left[C^{\prime}\right]=\sum_{p=1}^{r} d_{p}\left[C_{p}\right]$. Extending the notation in $\S 5.2$, write

$$
\begin{aligned}
c_{\tau}\left(C^{\prime}\right) & =c_{\tau}\left(\left.E\right|_{\left[C^{\prime}\right]}, \tau\right), \\
\mu_{\tau}\left(C^{\prime}\right) & =\sum_{i} \sum_{k=1}^{M_{i}} \mu_{\tau_{i}^{+}}\left(\alpha_{i}^{k}\right)-\sum_{j} \sum_{k=1}^{N_{j}} \mu_{\tau_{j}^{-}}\left(\beta_{j}^{k}\right), \\
Q_{\tau}\left(C^{\prime}\right) & =Q_{\tau}\left(\left[C^{\prime}\right],\left[C^{\prime}\right]\right) .
\end{aligned}
$$

For the rest of this proof, we drop the subscript ' $\tau$ '. By the definition of the relative index $I$,

$$
I\left(\left[C^{\prime}\right]\right)=c\left(C^{\prime}\right)+Q\left(C^{\prime}\right)+\mu\left(C^{\prime}\right) .
$$

Now $c$ is linear, and $Q$ is bilinear:

$$
\begin{aligned}
c\left(C^{\prime}\right) & =\sum_{p=1}^{r} d_{p} c\left(C_{p}\right), \\
Q\left(C^{\prime}\right) & =\sum_{p=1}^{r} d_{p}^{2} Q\left(C_{p}\right)+\sum_{p \neq p^{\prime}} d_{p} d_{p^{\prime}} Q\left(C_{p}, C_{p^{\prime}}\right) .
\end{aligned}
$$

By the index formula of Lemma 5.2 and our genericity assumption on $J$,

$$
\operatorname{dim}\left(\mathscr{M}_{C_{p}}\right) \leq c\left(C_{p}\right)+Q\left(C_{p}\right)+w\left(C_{p}\right)+\mu^{0}\left(C_{p}\right) .
$$

So by the above equations, our goal (66) is equivalent to

$$
\begin{aligned}
\sum_{p=1}^{r} d_{p}\left(w\left(C_{p}\right)+\mu^{0}\left(C_{p}\right)\right) \leq & \sum_{p=1}^{r}\left(d_{p}^{2}-d_{p}\right) Q\left(C_{p}\right) \\
& +\sum_{p \neq p^{\prime}} d_{p} d_{p^{\prime}} Q\left(C_{p}, C_{p^{\prime}}\right)+\mu\left(C^{\prime}\right) .
\end{aligned}
$$

We now eliminate $Q$ from this inequality. By Proposition 8.4 and Lemma 8.5,

$$
\begin{aligned}
Q\left(C_{p}\right) & \geq-w\left(C_{p}\right)-\eta\left(C_{p}\right), \\
Q\left(C_{p}, C_{p^{\prime}}\right) & =-\ell\left(C_{p}, C_{p^{\prime}}\right) .
\end{aligned}
$$

So to prove our goal (69), it suffices to show that

$$
\sum_{p=1}^{r}\left(d_{p}^{2} w\left(C_{p}\right)+\left(d_{p}^{2}-d_{p}\right) \eta\left(C_{p}\right)+d_{p} \mu^{0}\left(C_{p}\right)\right)+\sum_{p \neq p^{\prime}} d_{p} d_{p^{\prime}} \ell\left(C_{p}, C_{p^{\prime}}\right) \leq \mu\left(C^{\prime}\right) .
$$

To prove (70), it will suffice to prove the following two claims. First, fix a periodic orbit $\gamma$, and suppose that the flow line $C_{p}$ has incoming ends at $\gamma$ with 
multiplicities $q_{p, 1}, \ldots, q_{p, k_{p}}$ totalling $n_{p}$. Let $\xi_{p}$ denote the resulting braid. The first claim is that

$$
\begin{aligned}
\sum_{p=1}^{r}\left(d_{p}^{2} w\left(\xi_{p}\right)+\left(d_{p}^{2}-d_{p}\right)\right. & \left.\eta\left(\xi_{p}\right)+d_{p} \sum_{i=1}^{k_{p}} \mu\left(\gamma^{q_{p, i}}\right)\right)+ \\
+\sum_{p \neq p^{\prime}} d_{p} d_{p^{\prime}} \ell\left(\xi_{p}, \xi_{p^{\prime}}\right) & \geq \sum_{i=1}^{\sum_{p} d_{p} n_{p}} \mu\left(\gamma^{i}\right) .
\end{aligned}
$$

Note that since we are now restricting attention to a single periodic orbit, we are recycling the subscripts ' $i$ ' here and ' $j$ ' below. The second claim is that an analogue of (71) holds for outgoing ends. However by symmetry, as in the proof of Lemma 6.13, it will suffice to prove only the first claim (71).

To prove (71), define

$$
\rho_{p, i}=\left\lceil\frac{\mu\left(\gamma^{q_{p, i}}\right)}{2}\right\rceil .
$$

For $i=1, \ldots, k_{p}$, let $\eta_{p, i}$ denote the winding number with respect to $\tau$ of the $i^{t h}$ component of $\xi_{p}$. The winding bound of Lemma 6.6 gives

$$
\eta_{p, i} \geq \rho_{p, i} .
$$

To simplify notation, let us now combine the two indices $p, i$ into a single index $j$. That is, we let each pair $(p, i)$ correspond to some integer $j$, and we define $q_{j}=q_{p, i}$, $\rho_{j}=\rho_{p, i}$, and $d_{j}=d_{p}$. Using equation (59), Lemmas 6.8 and 6.9, and the inequality (72), we obtain

$$
\begin{aligned}
\sum_{p}\left(d_{p}^{2} w\left(\xi_{p}\right)+\left(d_{p}^{2}-d_{p}\right) \eta\left(\xi_{p}\right)\right) & +\sum_{p \neq p^{\prime}} d_{p} d_{p^{\prime}} \ell\left(\xi_{p}, \xi_{p^{\prime}}\right) \\
& \geq \sum_{j, j^{\prime}} d_{j} d_{j^{\prime}} \min \left(q_{j} \rho_{j^{\prime}}, q_{j^{\prime}} \rho_{j}\right)-\sum_{j} d_{j} \rho_{j} .
\end{aligned}
$$

Thus our goal (71) will follow from

$$
\sum_{j, j^{\prime}} d_{j} d_{j^{\prime}} \min \left(q_{j} \rho_{j^{\prime}}, q_{j^{\prime}} \rho_{j}\right)+\sum_{j} d_{j}\left(\mu\left(\gamma^{q_{j}}\right)-\rho_{j}\right) \geq \sum_{i=1}^{\sum_{j} d_{j} q_{j}} \mu\left(\gamma^{i}\right) .
$$

To prove (73), we simply apply the inequality of Lemma 6.10 to the tuple

$$
(\underbrace{q_{1}, \ldots, q_{1}}_{d_{1} \text { times }}, \ldots, \underbrace{q_{j}, \ldots, q_{j}}_{d_{j} \text { times }}, \ldots) .
$$

This completes the proof of Theorem 8.1 when the $C_{p}$ 's do not contain trivial cylinders. We now have to show that if $T$ is a union of trivial cylinders (possibly repeated), then $I\left(C^{\prime}\right) \leq I\left(C^{\prime}+T\right)$. The argument is a straightforward modification of the proof of the inequality of Proposition 7.1. 


\section{Compactness}

We now prove the compactness Theorem 1.8.

\subsection{Rigidity of trivial cylinders}

We begin with a useful basic fact.

Proposition 9.1 (rigidity). If $T$ is a union of trivial cylinders (possibly repeated), then $T$ is the only flow line in its relative homology class (ending at the same periodic orbits).

Proof. Let $u: C \rightarrow \mathbb{R} \times Y$ be a flow line homologous to $T$. Since $\int_{T} \omega=0$,

$$
\int_{C} u^{*} \omega=0
$$

By the definition of admissible almost complex structure, for any tangent vector $v \in T(\mathbb{R} \times Y)$ we have

$$
\begin{aligned}
\omega(v, J v) & \geq 0, \\
\omega(v, J v)=0 & \Longleftrightarrow v \in \operatorname{span}\left(\partial_{s}, \partial_{t}\right) .
\end{aligned}
$$

Since $u$ is a pseudoholomorphic immersion by the definition of flow line, it follows that it is locally a flat section of the bundle $\mathbb{R} \times Y \rightarrow \mathbb{R} \times S^{1}$. In other words, $u$ is a union of trivial cylinders (possibly repeated), so $u=T$.

Remark 9.2. A similar argument shows that in $S^{1} \times Y$, with an admissible almost complex structure, any embedded pseudoholomorphic curve in an $S^{1}$-invariant homology class is $S^{1}$ invariant. This fact is related to a result of Ionel and Parker [11], and can be used together with Taubes's "SW=Gr" theorem [19] to calculate the Seiberg-Witten invariants of $Y$, see [6].

\subsection{The case $d=1$, genus $(\Sigma)>0$}

If $d=1$, then any flow line is a pseudoholomorphic section of the bundle $\mathbb{R} \times Y \rightarrow$ $\mathbb{R} \times S^{1}$. The reason is that the fibers are pseudoholomorphic and have intersection number 1 with the flow line, and the flow line cannot contain a fiber or else it would not be embedded; so it follows from intersection positivity [14] that the flow line intersects each fiber transversely in a single point. If also genus $(\Sigma)>0$, then the compactness Theorem 1.8 is a special case of a standard result in Floer theory; the assumption that genus $(\Sigma)>0$ implies that $\pi_{2}(Y)=0$ so that there is no bubbling of pseudoholomorphic spheres.

In $§ 9.3-\S 9.4$ we will prove Theorem 1.8 when $\partial \Sigma \neq \emptyset$ or $d>\operatorname{genus}(\Sigma)$. 


\subsection{Generalized flow lines}

To understand limits of flow lines, we need to consider more general pseudoholomorphic curves which are not necessarily embedded.

Definition 9.3. A generalized flow line (GFL) from the orbit set $\left\{\left(\alpha_{i}, m_{i}\right)\right\}$ to the orbit set $\left\{\left(\beta_{j}, n_{j}\right)\right\}$ is a pseudoholomorphic map $u: C \rightarrow \mathbb{R} \times Y$, modulo reparametrization, where:

- $C$ is a punctured compact Riemann surface,

- $u$ has outgoing ends at $\alpha_{i}$ with total multiplicity $m_{i}$, incoming ends at $\beta_{j}$ with total multiplicity $n_{j}$, and no other ends.

We write $\chi(u)=\chi(C)$ and $[u]=u_{*}[C]$. We define the relative index $I(u)=$ $I(\alpha, \beta ;[u])$.

We say that $u$ is a quasi-embedding if $u$ is an embedding when restricted to the complement of a finite (possibly empty) set in $C$. If $u: C \rightarrow \mathbb{R} \times Y$ is any GFL and $(\phi, J)$ is admissible near its ends, then $u$ factors through a quasi-embedding $C^{\prime} \rightarrow \mathbb{R} \times Y$ via a branched covering $C \rightarrow C^{\prime}$, possibly the identity covering. The reason is that if $u$ has no multiply covered components, then $u$ is a quasiembedding, because the singularities are isolated [14], and the discussion in $\$ 6$ shows that there are no singularities for $|s|$ sufficiently large, where the intersection of $u(C)$ with $\{s\} \times Y$ is an iterated nested cabling of torus braids.

We say that $u$ is a connector if $u$ is a branched cover (possibly the identity) of a union of trivial cylinders. We say that a GL $u$ is nontrivial if $u$ is not a connector, or if $u$ is a connector with branch points.

Let $\widetilde{\mathscr{M}}_{u}$ denote the component of the moduli space of GFL's containing $u$. If $\tau$ is a trivialization of $E$ over the ends of $u$, we define the virtual dimension

$$
\operatorname{vir}-\operatorname{dim}\left(\tilde{\mathscr{M}}_{u}\right)=2 c_{\tau}(u)+\mu_{\tau}^{0}(u)-\chi(u) .
$$

Here $\mu_{\tau}^{0}$ is defined as in $§ 5.2$. The virtual dimension is a homotopy invariant of $u$. If $u$ is a quasi-embedding we let $\widehat{\mathscr{M}}_{u}$ denote the component of the moduli space of quasi-embedded GFL's containing $u$. If $(\phi, J)$ is admissible near the ends of $u$ and $J$ is generic, if $u$ is a quasi-embedding, and if $u$ does not contain any fibers of the projection $\mathbb{R} \times Y \rightarrow \mathbb{R} \times S^{1}$ then $\widehat{\mathscr{M}}_{u}$ is cut out transversely by Lemma 9.12(b). In this case we further have

$$
\operatorname{dim}\left(\widehat{\mathscr{M}}_{u}\right)=\operatorname{vir}-\operatorname{dim}\left(\widetilde{\mathscr{M}}_{u}\right) .
$$

This follows as in [3], or by a modification of the proof of Lemma 5.2.

Lemma 9.4 (parity of virtual dimension). If $\alpha$ and $\beta$ are admissible, and if $u$ is a GFL from $\alpha$ to $\beta$, then

$$
\operatorname{vir}-\operatorname{dim}\left(\widetilde{\mathscr{M}}_{u}\right) \equiv I(u) \bmod 2
$$


Proof. We have

$$
I(u)-\operatorname{vir}-\operatorname{dim}\left(\tilde{\mathscr{M}}_{u}\right)=-c_{\tau}([u])+Q_{\tau}([u],[u])+\mu_{\tau}(u)-\mu_{\tau}^{0}(u)+\chi(u) .
$$

Write $\alpha=\left\{\left(\alpha_{i}, m_{i}\right)\right\}$ and $\beta=\left\{\left(\beta_{j}, n_{j}\right)\right\}$. By equations (19) and (20),

$$
-c_{\tau}([u])+Q_{\tau}([u],[u]) \equiv \sum_{i} m_{i}-\sum_{j} n_{j} \bmod 2 .
$$

Let $k_{i}$ (resp. $l_{j}$ ) denote the number of ends of $u$ at $\alpha_{i}$ (resp. $\beta_{j}$ ). Then

$$
\chi(u) \equiv \sum_{i} k_{i}-\sum_{j} k_{j} \bmod 2 .
$$

Finally, one can see directly that since $\alpha$ and $\beta$ are admissible,

$$
\mu_{\tau}(u)-\mu_{\tau}^{0}(u) \equiv \sum_{i}\left(m_{i}-k_{i}\right)-\sum_{j}\left(n_{j}-l_{j}\right) \bmod 2 .
$$

Combining the above four equations proves the lemma.

Lemma 9.5 (low index GFL's). Let $\alpha$ and $\beta$ be orbit sets of degree $d$, and assume that $(\phi, J)$ is $d$-admissible and $J$ is generic. Let $u: C \rightarrow \mathbb{R} \times Y$ be a GFL from $\alpha$ to $\beta$. Assume that $\partial \Sigma \neq \emptyset$ or $d>\operatorname{genus}(\Sigma)$. Then:

(a) $I(u) \geq 0$.

(b) If $I(u)=0$, then $u$ is a connector.

(c) If $I(u)=1$, or $I(u)=2$ and $\alpha, \beta$ are admissible, then $u$ is the disjoint union of a nontrivial flow line and a (possibly empty) connector.

Proof. Let $C_{1}, \ldots, C_{r}$ be the components of the quasi-embedding $C^{\prime}$ above, regarded as subsets of $\mathbb{R} \times Y$, and let $d_{p}$ denote the covering multiplicity of $C$ over $C_{p}$. The projection $\mathbb{R} \times Y \rightarrow \mathbb{R} \times S^{1}$ restricts to a holomorphic map on each component $C_{p}$. Therefore:

(*) Each $C_{p}$ either has at least one incoming and one outgoing end, or is a fiber of the projection $\mathbb{R} \times Y \rightarrow \mathbb{R} \times S^{1}$.

We now consider two cases.

Case 1: Suppose that no $C_{p}$ is a fiber. Then

$$
\sum_{p=1}^{r} d_{p} \operatorname{dim}\left(\widehat{\mathscr{M}}_{C_{p}}\right) \leq I(u)-2 \Delta
$$

where $\Delta$ is a nonnegative integer which is zero only if the $C_{p}$ 's are embedded and disjoint. In fact, (77) holds with

$$
\Delta=\sum_{p} d_{p}^{2} \delta\left(C_{p}\right)+\sum_{p<p^{\prime}} d_{p} d_{p^{\prime}} \#\left(C_{p} \cap C_{p^{\prime}}\right) .
$$


The proof of (77) follows the proof of Theorem 8.1, except that we use equations (76), (75), and (18) in place of Lemma 5.2.

Assertion (a) now follows from (77). To prove (b), if $I(u)=0$, then $\operatorname{dim}\left(\widehat{\mathscr{M}}_{C_{p}}\right)$ $=0$ for each $p$ by (77), so $C_{p}$ is fixed under the translation action of $\mathbb{R}$ on $\mathbb{R} \times Y$, so $C_{p}$ is a union of trivial cylinders.

To prove (c), we first observe that if $I(u) \in\{1,2\}$, then the $C_{p}$ 's are embedded and disjoint, or else by (77) we would have $\operatorname{dim}\left(\widehat{\mathscr{M}}_{C_{p}}\right)=0$ so that the $C_{p}$ 's would be unions of trivial cylinders, giving $I(u)=0$.

Now suppose that $I(u)=1$. Then for some $p$, we have $\operatorname{dim}\left(\widehat{\mathscr{M}}_{C_{p}}\right)=d_{p}=1$. So $C_{p}$ is a nontrivial flow line; the other components of $u$ are connectors as in part (b), and they are disjoint from $C_{p}$ as explained above.

Finally suppose that $I(u)=2$ and $\alpha, \beta$ are admissible. We claim that if $C_{p}$ is nontrivial then $d_{p}=1$, so we are done as in the case $I(u)=1$. To prove the claim, suppose $d_{p}>1$. Since $\alpha$ and $\beta$ are admissible, all ends of $C_{p}$ are elliptic. It follows that $\operatorname{dim}\left(\widehat{\mathscr{M}}_{C_{p}}\right)$ is even, by equation (76), Lemma 9.4, and Proposition 1.6(c). By (77), $\operatorname{dim}\left(\widehat{\mathscr{M}}_{C_{p}}\right) \leq 1$, so we in fact have $\operatorname{dim}\left(\widehat{\mathscr{M}}_{C_{p}}\right)=0$. Therefore $C_{p}$ is a union of trivial cylinders.

Case 2: Suppose that some $C_{p}$ is a fiber of the projection $\mathbb{R} \times Y \rightarrow \mathbb{R} \times S^{1}$. Then $\partial \Sigma=\emptyset$, so by assumption $d>g=\operatorname{genus}(\Sigma)$. Let $u^{\prime}$ denote the GFL obtained from $u$ by deleting the component(s) covering $C_{p}$. By the index ambiguity formula of Proposition 1.6(d), we have

$$
I\left(u^{\prime}\right)=I(u)-2 d_{p}(d-g+1) \leq I(u)-4 .
$$

Thus removing all fibers from $u$ decreases the relative index by at least 4 . So by assertion (a) in Case 1, $I(u) \geq 4$. Thus assertion (a) holds in this case as well, while assertions (b) and (c) are vacuously true.

\subsection{Limits of sequences of flow lines}

To describe the possible limits of sequences of flow lines, we need the following definitions.

Definition 9.6. A $k$-times broken GFL from $\alpha$ to $\beta$ is a sequence $\left(u_{1}, \ldots, u_{k}\right)$, where there exist orbit sets $\alpha_{0}, \ldots, \alpha_{k}$ such that $u_{i}$ is a nontrivial GFL from $\alpha_{i-1}$ to $\alpha_{i}$, and $\alpha_{0}=\alpha$ and $\alpha_{k}=\beta$. We also assume that at each periodic orbit in $\alpha_{i}$, the multiplicities of the incoming ends of $u_{i}$ agree with the multiplicities of the outgoing ends of $u_{i+1}$.

If $\psi \in \mathbb{R}$, let $T_{\psi}: \mathbb{R} \times Y \rightarrow \mathbb{R} \times Y$ denote the translation sending $(s, y) \mapsto$ $(s-\psi, y)$.

Definition 9.7. Let $\left(u_{1}, \ldots, u_{k}\right)$ be a broken GFL from $\alpha$ to $\beta$. Let $\left\{v_{1}, v_{2}, \ldots\right\}$ be a sequence of GFL's from $\alpha$ to $\beta$ in a fixed relative homology class $Z$ whose 
domains have a fixed topological type. We say that $\left\{v_{n}\right\}$ converges to $\left(u_{1}, \ldots, u_{k}\right)$ if:

(a) The domain of $v_{n}$ converges in Deligne-Mumford space (possibly after adding some marked points to the $v_{n}$ 's and $u_{i}$ 's) to a nodal curve which can be decomposed into a union of curves $C_{1}, \ldots, C_{k}$, such that $C_{i}$ intersects $C_{i+1}$ in one node for each incoming end of $u_{i}$ and $C_{i-1}$ in one node for each outgoing end of $u_{i}$; and after puncturing $C_{i}$ at these points, we obtain the domain of $u_{i}$, modulo identification of points with the same image in $(b)$.

(b) There are real numbers $s_{1, n}>s_{2, n}>\cdots>s_{k, n}$ for each positive integer $n$ such that $T_{s_{i, n}} \circ v_{n} \rightarrow u_{i}$ in $C^{\infty}$ on compact sets.

(c) $\sum_{i=1}^{k}\left[u_{i}\right]=Z$.

We now have the following version of Gromov compactness. If $u: C \rightarrow \mathbb{R} \times Y$ is a pseudoholomorphic map, we define the energy $E(u)=\int_{C} u^{*} \omega$.

Lemma 9.8 (general compactness). Let $\left\{C_{n}\right\}_{n=1,2, \ldots}$ be a sequence of flow lines from $\alpha$ to $\beta$, with the energy $E\left(C_{n}\right)$ uniformly bounded from above. Assume that $(\phi, J)$ is admissible near the periodic orbits in $\alpha$ and $\beta$. Then there is a subsequence with a fixed relative homology class and topological type which converges in the above sense to a broken GFL $\left(u_{1}, \ldots, u_{k}\right)$.

The proof of Lemma 9.8 is mostly a standard argument using Gromov compactness, except that we need to be careful because the genus of $C_{n}$ is not a priori bounded. Fortunately, in dimension four a version of Gromov compactness is available using currents which does not assume any such bound $[21,23]$. We will use the following special case of it.

Lemma 9.9 (currents). Fix $d \in \mathbb{Z}$ and $E_{0} \in \mathbb{R}$. Let $u_{k}: C_{k} \rightarrow \mathbb{R} \times Y$ be a sequence of proper pseudoholomorphic maps such that for each $k$, the current $u_{k}\left[C_{k}\right]$ is a homology between two orbit sets of degree $d$, and $E\left(u_{k}\right)<E_{0}$. Then we can pass to a subsequence such that:

- The $u_{k}$ 's converge weakly as currents in $\mathbb{R} \times Y$ to a proper pseudoholomorphic map $u: C \rightarrow \mathbb{R} \times Y$.

- For any compact set $K \subset \mathbb{R} \times Y$, as $k \rightarrow \infty$,

$$
\sup _{x \in u_{k}\left(C_{k}\right) \cap K} \operatorname{dist}(x, u(C))+\sup _{x \in u(C) \cap K} \operatorname{dist}\left(x, u_{k}\left(C_{k}\right)\right) \rightarrow 0 .
$$

Proof. We first note that there is a natural symplectic form

$$
\Omega=\omega+d s \wedge d t
$$

on $\mathbb{R} \times Y$. An admissible almost complex structure $J$ on $Y$ is tamed by $\Omega$ :

$$
\Omega(v, J v)>0 \text { for } v \neq 0 \text {. }
$$


Furthermore, $\mathbb{R} \times Y$ is exhausted by compact sets $[a, b] \times Y$, on each of which we have a uniform bound

$$
\int_{u_{k}\left[C_{k}\right] \cap([a, b] \times Y)} \Omega \leq d(b-a)+E_{0}
$$

by (74). The lemma now follows as in [21, Prop. 3.3] or [23].

Proof of Lemma 9.8. We proceed in 4 steps. As usual let $d$ denote the degree of $\alpha$ and $\beta$. We choose a metric on $Y$, and work with the product metric on $\mathbb{R} \times Y$. We can pass to a subsequence such that $E\left(C_{n}\right) \rightarrow E_{0}$. Let $\Gamma_{d}$ denote the union of the periodic orbits of period $\leq d$.

Step 1: We claim that for any sequence of real numbers $s_{n}$, we can pass to a subsequence such that $T_{S_{n}}\left(C_{n}\right)$ converges in the sense of Lemma 9.9 to a GFL.

To prove this, we can pass to a subsequence such that $T_{s_{n}}\left(C_{n}\right)$ converges in the sense of Lemma 9.9 to some proper pseudoholomorphic map $u: C \rightarrow \mathbb{R} \times Y$. Since the integral of $u^{*} \omega$ is locally nonnegative, using the weak convergence of currents we obtain $E(u) \leq E_{0}$.

Now $u(C)$ is $C^{0}$-asymptotic to a union of trivial cylinders as $s \rightarrow+\infty$. Otherwise there exists $\epsilon>0$ and a sequence $\psi_{k} \rightarrow \infty$ such that $u(C) \cap\left(\left\{\psi_{k}\right\} \times Y\right)$ contains a point of distance greater than $\epsilon$ from $\mathbb{R} \times \Gamma_{d}$. The sequence of translates $T_{\psi_{k}} \circ u$ contains a subsequence converging in the sense of Lemma 9.9 to some pseudoholomorphic curve. Since $u$ has finite energy and $\psi_{k} \rightarrow \infty$, the limiting curve has energy zero and hence its image is a union of trivial cylinders as in Proposition 9.1. Together with (78), this contradicts our distance assumption. Likewise, $u(C)$ is $C^{0}$-asymptotic to a possibly different union of trivial cylinders as $s \rightarrow-\infty$.

We will see below, using the assumption that the $C_{n}$ 's are flow lines, that we can pass to a further subsequence so that the topological type of $C_{n}$ is fixed. Then the domain $C$ is a punctured compact Riemann surface, and by standard lemmas we have $C^{\infty}$ convergence to trivial cylinders at the punctures, so $u$ is a GFL.

Step 2: Suppose $u: C \rightarrow \mathbb{R} \times Y$ is a proper pseudoholomorphic map such that $\overline{u[C]}$ is a homology between orbit sets of degree $d$, and suppose $u(C)$ is not a union of trivial cylinders. Then we claim that there is a constant $\delta>0$ depending only on $d$ such that $E(u)>\delta$.

If not, then we can take a sequence $v_{k}: C_{k}^{\prime} \rightarrow \mathbb{R} \times Y$ of such maps with $E\left(v_{k}\right) \rightarrow 0$. Let $N$ denote the set of points within distance $\epsilon$ of $\Gamma_{d}$, where $\epsilon>0$ is chosen small enough so that $N$ is a tubular neighborhood of $\Gamma_{d}$. Since $v_{k}\left(C_{k}^{\prime}\right)$ is not a union of trivial cylinders, $E\left(v_{k}\right)>0$ by equation (74), so on homological grounds $v_{k}\left(C_{k}^{\prime}\right) \not \subset \mathbb{R} \times N$. By applying translations we may assume that $v_{k}\left(C_{k}^{\prime}\right) \cap(\{0\} \times Y)$ contains a point of distance at least $\epsilon$ from $\mathbb{R} \times \Gamma_{d}$. Applying Lemma 9.9 as in the third paragraph of Step 1 gives a contradiction.

Step 3: We now apply Step 1 with judicious choices of $s_{n}$. We can assume that $C_{n}$ is not a union of trivial cylinders for large $n$, or else the lemma is trivially true. We can then define

$$
s_{1, n}=\sup \left\{s \in \mathbb{R} \mid C_{n} \cap(\{s\} \times Y) \not \subset\{s\} \times N\right\} .
$$


By Step 1 , we can pass to a subsequence so that $T_{S_{1, n}}\left(C_{n}\right)$ converges to a GFL $u_{1}$, whose image is not a union of trivial cylinders, with $E\left(u_{1}\right) \leq E_{0}$.

Suppose $E\left(u_{1}\right)<E_{0}$. Since $u_{1}$ is asymptotic to some union of periodic orbits of period $\leq d$ as $s \rightarrow-\infty$, we can find $\psi_{1} \in \mathbb{R}$ such that $\operatorname{dist}\left(y, \Gamma_{d}\right)<\epsilon / 2$ whenever $y \in Y,(s, y) \in \operatorname{Im}\left(u_{1}\right)$, and $s<\psi_{1}$. Then for large $n, C_{n} \cap\left(\left(-\infty, s_{1, n}+\psi_{1}\right) \times Y\right)$ is not contained in $\mathbb{R} \times N$, or else $C_{n}$ would be homologous to $u_{1}$ and would have the same energy. We can then define

$$
s_{2, n}=\sup \left\{s<s_{1, n}+\psi_{1} \mid C_{n} \cap(\{s\} \times Y) \not \subset\{s\} \times N\right\} .
$$

By Step 1, we can pass to a subsequence so that $T_{S_{2, n}}\left(C_{n}\right)$ converges to a GFL $u_{2}$. Continuing this process, suppose that $s_{i, n}$ have been chosen for $i<k$. If $E\left(u_{1}\right)+\cdots+E\left(u_{k-1}\right)<E_{0}$, we define $\psi_{k-1}$ from $u_{k-1}$ as above, set

$$
s_{k, n}=\sup \left\{s<s_{k-1, n}+\psi_{k-1} \mid C_{n} \cap(\{s\} \times Y) \not \subset\{s\} \times N\right\},
$$

and pass to a subsequence so that $T_{s_{k, n}}\left(C_{n}\right)$ converges to a GFL $u_{k}$.

This process must eventually stop since the sum of the $E\left(u_{i}\right)$ 's is bounded from above by $E_{0}$, while by Step 2 each $E\left(u_{i}\right)$ is bounded from below by $\delta>0$. We obtain GFL's $u_{1}, \ldots, u_{k}$ and real numbers $s_{1, n}>s_{2, n}>\cdots>s_{k, n}$ for each $n$ such that the relative homology class $\left[C_{n}\right]$ is eventually constant and equals $\sum_{i}\left[u_{i}\right]$, and $T_{s_{i, n}}\left(C_{n}\right) \rightarrow u_{i}$ in the sense of Lemma 9.9.

Step 4: We can pass to a subsequence so that the relative homology class $\left[C_{n}\right]$ is fixed. By Theorem 10.1(a), there is a lower bound on the Euler characteristic $\chi\left(C_{n}\right)$. This implies that we can pass to a subsequence so that the topological type of $C_{n}$ is fixed. As in [4], we can then obtain convergence in the sense of Definition 9.7, by passing to a further subsequence and possibly inserting some nontrivial connectors in between $u_{1}, \ldots, u_{k}$ to obtain the limiting broken GFL.

Together with Lemma 9.8, the following lemma will complete the proof of Theorem 1.8(a).

Lemma 9.10 (possible limits). Let $\alpha$ and $\beta$ be orbit sets of degree $d$. Let $\left(u_{1}, \ldots, u_{k}\right)$ be a broken GFL from $\alpha$ to $\beta$ which is the limit of a sequence of flow lines $\left\{C_{n}\right\}$ in the same relative homology class of relative index $I_{0} \in\{1,2\}$. Assume that $\partial \Sigma \neq \varnothing$ or $d>\operatorname{genus}(\Sigma)$. Suppose $(\phi, J)$ is $d$-admissible and $J$ is generic. Then:

(a) If $I_{0}=1$, then $k=1$ and $u_{1}$ is a nontrivial flow line.

(b) If $I_{0}=2$ and $\alpha, \beta$ are admissible, then $u_{1}$ and $u_{k}$ are nontrivial flow lines, and $u_{i}$ is a nontrivial connector for $1<i<k$.

Proof. Assume that (a) $I_{0}=1$, or (b) $I_{0}=2$ and $\alpha, \beta$ are admissible.

Step 1: By the additivity of the relative index in Proposition 1.6(b), we have $\overline{\sum_{i=1}^{k} I} I\left(u_{i}\right)=I_{0}$. By Lemma 9.5, it follows that each $u_{i}$ is either a nontrivial connector, or the disjoint union of a nontrivial flow line and a (possibly empty) connector.

Step 2: The GFL's $u_{1}, \ldots, u_{k}$ cannot all be connectors, or else $I\left(C_{n}\right)=0$. 
Step 3: A connector lives in a moduli space of nonnegative virtual dimension, see $\overline{\text { Step } 5}$. A nontrivial flow line lives in a moduli space of positive virtual dimension, by equation (76) and the $\mathbb{R}$-action. Also, by (75) and Definition 9.7,

$$
\sum_{i=1}^{k} \operatorname{vir-\operatorname {dim}}\left(\widetilde{\mathscr{M}}_{u_{i}}\right) \leq \lim _{n \rightarrow \infty} \operatorname{vir-\operatorname {dim}}\left(\tilde{\mathscr{M}}_{C_{n}}\right) \leq I_{0} .
$$

The inequality on the right holds by the relative adjunction formulas and the inequality (30).

Step 4: The $C_{n}$ 's are admissible. Otherwise, if some $C_{n}$ is not admissible, then $\overline{\operatorname{vir}-\operatorname{dim}}\left(\widetilde{\mathscr{M}}_{C_{n}}\right)<I_{0}$ as in Theorem 1.7, and moreover in case (b) the difference is at least two by Lemma 9.4. Consequently $\operatorname{dim}\left(\widehat{\mathscr{M}}_{C_{n}}\right)=0$ by (76), so $C_{n}$ is a union of trivial cylinders, so $I\left(C_{n}\right)=0$, a contradiction.

Step 5: $u_{1}$ and $u_{k}$ do not contain any nontrivial connectors.

Otherwise, WLOG $u_{k}$ contains a nontrivial connector $v$. Then vir- $\operatorname{dim}\left(\widetilde{\mathscr{M}}_{v}\right) \geq 1$, and in case (b) vir-dim $\left(\widetilde{\mathscr{M}}_{v}\right) \geq 2$. The reason is that we can compute vir-dim( $\left(\widetilde{\mathscr{M}}_{v}\right)$ from equation (75) by starting at the bottom of $v$ and moving vertically past one branch point at a time. By homotopy invariance of virtual dimension, we can assume that the branch points are simple. Then each time we move past a branch point, two circles in $v$ join into one or one circle separates into two. By Proposition 2.1, the contribution to the virtual dimension is 1 for hyperbolic orbits, and 0 or 2 for elliptic orbits. If the branching is over an elliptic orbit, then the bottom branch point contributes 2 to the virtual dimension, by Step 4 and Lemma 4.5(b),(c). In case (b), the branching is always over an elliptic orbit, since $\alpha$ and $\beta$ are admissible.

By Step 3, all other components of the $u_{i}$ 's live in moduli spaces of virtual dimension 0 , so no component of any $u_{i}$ is a nontrivial flow line. But this contradicts Steps 1 and 2.

Step 6: Now we are done. By Steps 1 and 5, $u_{1}$ and $u_{k}$ are nontrivial flow lines. By $\overline{\text { Step } 3}, u_{i}$ is a nontrivial connector for $1<i<k$. If $I_{0}=1$, then we must have $k=1$ by additivity of the relative index.

\subsection{Fiber bubbles and transversality}

The proof of Theorem 1.8(a) is now complete. We now prove Theorem 1.8(b). By Theorem 1.8(a), we can assume that $\partial \Sigma=\varnothing$ and $1<d \leq g$. In this case, compactness might not hold for an admissible almost complex structure, because the fibers of the projection $\mathbb{R} \times Y \rightarrow \mathbb{R} \times S^{1}$ are pseudoholomorphic and might bubble off. To ensure compactness, we need to relax the requirement that the almost complex structure preserve $E$.

Definition 9.11. Let $J$ be an almost complex structure on $\mathbb{R} \times Y$. We say that $(\phi, J)$ is almost $d$-admissible if:

(a) $J$ is $\mathbb{R}$-invariant.

(b) $J\left(\partial_{s}\right)=\partial_{t}$. 
(c) There is an almost complex structure $J^{\prime}$ such that $\left(\phi, J^{\prime}\right)$ is d-admissible and $J$ agrees with $J^{\prime}$ on $\mathbb{R}$ cross a neighborhood of the periodic orbits of period $\leq d$ and a neighborhood of $\partial Y$.

(d) J is $\Omega$-tame, i.e. equation (79) holds.

Of course, if $J$ is $d$-admissible, then $J$ is almost $d$-admissible. Also note that if $J$ is almost $d$-admissible, then a $C^{0}$-small perturbation (with respect to an $\mathbb{R}$-invariant metric on $\mathbb{R} \times Y$ ) of $J$ satisfying conditions (a), (b), and (c) will automatically satisfy the taming condition (d).

Condition (b) implies that $J$ preserves a 2-plane bundle $E^{\prime} \subset T Y$, and (b) and (d) imply that $E^{\prime}$ is homotopic to $E$. This implies that the relative adjunction formula (12) still holds.

Condition (b) implies that (74) still holds, so Proposition 9.1 still holds.

Lemma 9.12 (transversality). Let $C$ be a quasi-embedded GFL and let $d$ be any positive integer. Then $\widehat{\mathscr{M}}_{C}$ is cut out transversely, provided that either:

(a) $(\phi, J)$ is almost d-admissible and $J$ is generic, or

(b) $(\phi, J)$ is $d$-admissible and $J$ is generic, and $C$ contains no fibers of the projection $\mathbb{R} \times Y \rightarrow \mathbb{R} \times S^{1}$.

Proof. (a) We can assume that our GFL's contain no trivial cylinders, as these are always cut out transversely. The proof is now a slight modification of the proof of [5, Thm. 5.1(i)]. The essential point is the following:

Claim. For any almost $d$-admissible $(\phi, J)$ and any quasi-embedded GFL $C$ without trivial cylinders, there is a nonempty open set $U \subset C$ away from a neighborhood of the period $\leq d$ periodic orbits, such that for each $x \in U$ :

(i) $\pi^{-1}(\pi(x))=\{x\}$, where $\pi: C \rightarrow Y$ denotes the projection.

(ii) $C$ is nonsingular at $x$, and the derivative of $\bar{\partial}_{J}(C)$ with respect to $J$, namely the bundle map

$$
\left\{\psi \in \operatorname{Hom}_{J}^{0,1}\left(\left.T X\right|_{C},\left.T X\right|_{C}\right)|\psi|_{\operatorname{span}\left(\partial_{s}, \partial_{t}\right)}=0\right\} \longrightarrow \operatorname{Hom}_{J}^{0,1}\left(T C, N_{C}\right),
$$

defined by restricting $\psi$ to $T C$ and projecting to $N_{C}$, is surjective at $x$. Here $\operatorname{Hom}_{J}^{0,1}$ denotes the space of $J$-antilinear maps.

To prove the claim, we will in fact obtain properties (i) and (ii) on an open dense set $U$ in $C$. Condition (i) holds on a dense open set just as in [10, Thm. 1.13]. Regarding (ii), we observe that with respect to $J$, the bundle map (80) is complex linear. Since the bundle $\operatorname{Hom}_{J}^{0,1}\left(T C, N_{C}\right)$ has complex rank one, the map (80) is surjective except where it is zero. But it is zero only where $C$ is tangent to $\operatorname{span}\left(\partial_{s}, \partial_{t}\right)$. The set of points where $C$ is not tangent to $\operatorname{span}\left(\partial_{s}, \partial_{t}\right)$ is certainly open, and it is also dense, or else by a unique continuation argument $C$ would contain a trivial cylinder.

(b) We first note that if $C$ does not contain a fiber, then no GFL in $\widehat{\mathscr{M}}_{C}$ contains a fiber, because any pseudoholomorphic curve close to a fiber is also a fiber, as the projection $\mathbb{R} \times Y \rightarrow \mathbb{R} \times S^{1}$ is holomorphic. The proof of transversality is now 
similar to part (a). In this case we assume that $C$ contains no trivial cylinders and fibers; and we show that on an open dense set $U \subset C$, for $x \in U$, conditions (i) and (ii) above hold, but with the map (80) replaced by the map

$$
\operatorname{Hom}_{J}^{0,1}\left(\left.E\right|_{C},\left.E\right|_{C}\right) \longrightarrow \operatorname{Hom}_{J}^{0,1}\left(T C, N_{C}\right)
$$

that sends $\psi \in \operatorname{Hom}_{J}^{0,1}\left(\left.E\right|_{C},\left.E\right|_{C}\right)$ to the composition

$$
\left.\left.\left.T C \longrightarrow E\right|_{C} \stackrel{\psi}{\longrightarrow} E\right|_{C} \longrightarrow N\right|_{C} .
$$

Here the left and right arrows are projections. The map (81) is surjective at $x$ unless $T_{x} C$ agrees with $\operatorname{span}\left(\partial_{s}, \partial_{t}\right)$ or $E_{x}$. Thus, by a unique continuation argument, the map (81) is surjective on an open dense set in $C$ as long as $C$ does not contain a fiber or a trivial cylinder.

Lemma 9.13. If $(\phi, J)$ is almost $d$-admissible and $J$ is generic, then Theorem 1.7 still holds, and the inequality (77) holds for any quasi-embedded GFL's $C_{1}, \ldots, C_{r}$.

Proof. All necessary changes to the proofs are handled by Lemma 9.12(a) and the remarks preceding it. The rest of the proofs of Theorem 1.7 and the inequality (77) either do not involve $J$, or are local to the periodic orbits of period $\leq d$ and hence are unchanged by condition (c) in Definition 9.11.

Lemma 9.14. There exists an almost $d$-admissible $(\phi, J)$ such that $J$ is generic as in Lemma 9.12(a), for which the analogue of Lemma 9.5 holds, assuming only $g>1$ instead of $\partial \Sigma \neq \emptyset$ or $d>\operatorname{genus}(\Sigma)$.

Proof. We have to modify the proof of Lemma 9.5 in two ways.

First, statement (*) in that proof is not necessarily true, as the projection $\mathbb{R} \times Y \rightarrow \mathbb{R} \times S^{1}$ may no longer be pseudoholomorphic. Nonetheless, there exists almost $d$-admissible $(\phi, J)$ with $J$ generic such that instead we have:

${ }^{\left(*^{\prime}\right)}$ Each $C_{p}$ either has at least one incoming and one outgoing end, or is homologous to a positive multiple of the class of the fiber of the projection $\mathbb{R} \times Y \rightarrow \mathbb{R} \times S^{1}$

If not, then we can take a sequence of generic $J_{n}$ with $\left(\phi, J_{n}\right)$ almost $d$-admissible, converging to a generic $J_{\infty}$ such that $\left(\phi, J_{\infty}\right)$ is $d$-admissible, together with $J_{n^{-}}$ pseudoholomorphic curves that do not satisfy $\left({ }^{\prime}\right)$. By Gromov compactness, a subsequence of these curves converges to a $J_{\infty}$-holomorphic curve which by ${ }^{*}$ ) cannot exist.

Second, we have to show that $C_{p}$ cannot to be homologous to $k$ times the fiber class. If it is, then using equation (18) and our assumption that $g>1$, we have

$$
\operatorname{vir}-\operatorname{dim}\left(\widehat{\mathscr{M}}_{C_{p}}\right)=k(2-2 g)-2 \delta\left(C_{p}\right)<0 .
$$

If $J$ is generic, then this is impossible by Lemma 9.12(a).

For the $J$ given by Lemma 9.14, the rest of $\S 9.3$ and $\S 9.4$ carries over unchanged, and so the proof of Theorem 1.8(b) is now complete. 


\section{The Euler characteristic of flow lines}

The following theorem is useful for determining what the flow lines can look like in specific examples. We use the notation of $\$ 5.2$.

Theorem 10.1 (Euler characteristic). Let $C$ be a flow line without trivial cylinders such that $(\phi, J)$ is admissible near its ends, and let $\tau$ be any trivialization of E over the ends. Then:

(a) $\chi(C) \geq c_{\tau}(C)-\mu_{\tau}(C)+\mu_{\tau}^{0}(C)-Q_{\tau}(C)$.

(b) If $J$ is generic and $I(C)=1$, then equality holds in $(a)$.

Proof. (a) The inequality follows by combining the relative adjunction formulas (12) and (13) and the inequality (30).

(b) If $J$ is generic, then by the relative adjunction formulas and Lemma 5.2,

$$
\chi(C)-c_{\tau}(C)+\mu_{\tau}(C)-\mu_{\tau}^{0}(C)+Q_{\tau}(C) \leq I(C)-\operatorname{dim}\left(\mathscr{M}_{C}\right) .
$$

If $I(C)=1$, then since $C$ is not a union of trivial cylinders, $\mathbb{R}$ acts nontrivially on $C$ by translation on $\mathbb{R} \times Y$, so $\operatorname{dim}\left(\mathscr{M}_{C}\right) \geq 1$, so equality holds in (a).

\section{Concluding remarks}

1. The combinatorics in the proof of the index inequality (3) are delicate, because the inequality is sharp, at least in the following sense: If $C$ is an admissible flow line without trivial cylinders, and if the leading coefficient in the asymptotic expansion of each end is nonzero, cf. equation (33), then equality holds. Some key points in proving this are as follows. The condition on the leading coefficients implies that equality holds in the winding bound (35). Admissibility then implies that equality also holds in the inequalities (36) and (43), because $\operatorname{gcd}\left(\left\lceil\mu_{\tau}\left(\gamma^{q}\right) / 2\right\rceil, q\right)=1$ for $q \in p_{\text {in }}(\gamma, n)$. Equality then holds in Lemma 6.12; admissibility implies that equality holds in Lemma 6.10 where it is applied. Likewise equality holds in Lemma 6.13.

2. For the dimensions of moduli spaces of quasi-embeddings that are not embeddings, an analogue of the index inequality (3) holds and is strict, by equation (77) with $r=d_{1}=1$, as long as we assume (a) or (b) in Lemma 9.12 to ensure transversality. Roughly speaking, smoothing a singularity increases the dimension of the moduli space by a positive even integer.

3 . If we replace $\Sigma$ with a symplectic manifold of dimension greater than 2 , then one cannot in general bound the dimension of a moduli space of embedded curves from above by an additive relative index defined only in terms of the ends of the flow lines and their relative homology class.

4. In this paper we studied the mapping torus $Y$, the periodic orbits of $\phi$, and the symplectic 4-manifold $(\mathbb{R} \times Y, \omega+d s \wedge d t)$. One could instead consider a 3-manifold $Y$ with a contact 1 -form $\alpha$, the closed orbits of the associated Reeb flow, and the symplectization $\left(\mathbb{R} \times Y, d\left(e^{s} \alpha\right)\right)$. One can consider embedded pseudoholomorphic curves in the symplectization with ends asymptotic to Reeb orbits [3]. One can 
state a formal analogue of our index inequality (3), and we conjecture that it is true. The main issue in proving it, at least following our approach, would be to better understand the asymptotic behavior of the ends of flow lines. (Or one might avoid this issue by perturbing the setup near short Reeb orbits as in §1.3.) One should then further be able to obtain analogues of our compactness results; the fiber bubbling which can happen for mapping tori has no analogue in this setting.

Acknowledgements. It is a pleasure to thank Y. Eliashberg, D. Salamon, M. Thaddeus and C. Young for many invaluable discussions; the ETH Zürich, the Institute for Theoretical Physics in Santa Barbara, and the Max Planck Institute for Mathematics in Bonn for their hospitality while parts of this work were done; and the National Science Foundation for partial financial support.

\section{References}

1. C. Abbas: Finite energy surfaces and the chord problem. Duke Math. J. 96(2), 241-316 (1999)

2. R. Carter, G. Segal, I. Macdonald: Lectures on Lie groups and Lie algebras. Cambridge: London Math. Soc. Student Texts 32 (1995)

3. Ya. Eliashberg, A. Givental, H. Hofer: Introduction to symplectic field theory. Published in GAFA (2000)

4. Ya. Eliashberg, H. Hofer, K. Wysocki, E. Zehnder: Symplectic field theory I. Compactness. In preparation

5. A. Floer, H. Hofer, D. Salamon: Transversality in elliptic Morse theory for the symplectic action. Duke Math. J. 80(1), 251-292 (1995)

6. M. Hutchings, Y-J. Lee: Circle-valued Morse theory, Reidemeister torsion, and SeibergWitten invariants of 3-manifolds. Topology 38(4), 861-888 (1999)

7. M. Hutchings, M. Thaddeus: Periodic Floer homology. In preparation

8. H. Hofer, K. Wysocki, E. Zehnder: Properties of pseudoholomorphic curves in symplectisations. I. Asymptotics. Ann. Inst. H. Poincaré Anal. Non Linéaire 13(3), 337-379 (1996). (Correction in Ann. Inst. H. Poincaré Anal. Non Linéaire 15(4), 535-538 (1998))

9. H. Hofer, K. Wysocki, E. Zehnder: Properties of pseudoholomorphic curves in symplectisations. II. Embedding controls and algebraic invariants. Geom. and Func. Anal. $\mathbf{5}(2), 270-328(1995)$

10. H. Hofer, K. Wysocki, E. Zehnder: Properties of pseudoholomorphic curves in symplectizations. III. Fredholm theory. Topics in nonlinear analysis, 381-475. Progr. Nonlinear Differential Equations Appl., 35, Basel: Birkhäuser 1999

11. E. Ionel, T. Parker: Gromov invariants and symplectic maps. Math. Ann. 314(1), 127158 (1999)

12. E. Ionel, T. Parker: The symplectic sum formula for Gromov-Witten invariants. math.SG/0010217

13. T. Kato: Perturbation theory for linear operators. Classics in Mathematics reprint, Springer 1995

14. D. McDuff: Singularities and positivity of intersections of $J$-holomorphic curves. In: M. Audin, F. Lafontaine, (eds.): Holomorphic curves in symplectic geometry, pp. 191216. Progress in Mathematics 117, Birkhäuser 1994

15. D. McDuff, D. Salamon: J-holomorphic curves and quantum cohomology. University Lecture Series 6, Amer. Math. Soc. 1994

16. D. Salamon: Seiberg-Witten invariants of mapping tori, symplectic fixed points, and Lefschetz numbers. Proceedings of 6th Gökova Geometry-Topology Conference. Turkish J. Math. 23(1), 117-143 (1999)

17. D. Salamon, E. Zehnder: Morse theory for periodic solutions of Hamiltonian systems and the Maslov index. Comm. Pure Appl. Math. 45(10), 1303-1360 (1992) 
18. M. Schwarz: Cohomology operations from $S^{1}$-cobordisms in Floer homology. $\mathrm{PhD}$ thesis, ETH Zürich 1995

19. C.H. Taubes: The Seiberg-Witten and Gromov invariants. Math. Res. Lett. 2(2), 221238 (1995)

20. C.H. Taubes: Counting pseudo-holomorphic submanifolds in dimension four. J. Differential Geom. 44(4), 818-893 (1996)

21. C.H. Taubes: The structure of pseudoholomorphic subvarieties for a degenerate almost complex structure and symplectic form on $S^{1} \times B^{3}$. Geom. Topol. 2, 221-332 (1998)

22. C.H. Taubes: The Seiberg-Witten invariants and 4-manifolds with essential tori. Geom. Topol. 5, 441-519 (2001)

23. C. Young: A new proof of Gromov compactness in dimension 4. In preparation 